\title{
Dingwiederholungen in Wolframs Parzival
}

\section{Einleitung}

„Wiederholung ist kein prominenter Begriff der Erzähltheorie.“1 Diese resignative Aussage von Rainer Warning aus den Jahren 2001 bzw. 2003 ist inzwischen namentlich durch Forschungen zur mittelalterlichen Literatur, zu denen Warning selbst beigetragen hat, ${ }^{2}$ überholt. Sie war es bis zu einem gewissen Grad schon 2001/03, wenn man den Begriff der Wiederholung weit fasst und ihm das Wiedererzählen subsumiert. Franz Josef Worstbrock, sein Schülerkreis und weitere Forschende haben aufgezeigt, welch wichtige Funktion das Wiedererzählen in der mittelalterlichen Literatur, besonders in der Epik einnimmt. ${ }^{3}$ Dabei kamen Formen der Aneignung und - im weiteren Sinn - der Übersetzung bzw. Übertragung von Erzählstoffen in den Blick, die zuvor mit dem umstrittenen Begriff der Adaptation courtoise $^{4}$ verbunden waren. Der Begriff des Wiedererzählens bezog sich aber auch auf Binnenstrukturen in den einzelnen Erzählungen, auf Formen der Wiederholung, die an Phänomene wie die bele conjointure und den doppelten Cursus geknüpft sind. ${ }^{5}$

1 Rainer Warning: Erzählen im Paradigma. Kontingenzbewältigung und Kontingenzexposition. In: Romanistisches Jahrbuch 52 (2001), S. 176-209, hier S. 176; ders.: Die narrative Lust an der List. Norm und Transgression im Tristan. In: Transgressionen. Literatur als Ethnographie. Hrsg. von Gerhard Neumann, Rainer Warning. Freiburg i. Breisgau 2003 (Rombach Wissenschaften. Reihe Litterae. 98), S. 175-212, hier S. 179.

2 Vgl. z. B. Rainer Warning: Fiktion und Transgression. In: Fiktion und Fiktionalität in den Literaturen des Mittelalters. Jan-Dirk Müller zum 65. Geburtstag. Hrsg. von Ursula Peters, Rainer Warning. München 2009, S. 31-55, bes. S. $46 \mathrm{f}$.

3 Vgl. stellvertretend Franz Josef Worstbrock: Wiedererzählen und Übersetzen. In: Mittelalter und frühe Neuzeit. Übergänge, Umbrüche und Neuansätze. Hrsg. von Walter Haug. Tübingen 1999 (Fortuna vitrea. 16), S. 128-142. Nachdruck in: ders.: Ausgewählte Schriften. Hrsg. von Susanne Köbele, Andreas Kraß. Bd. 1: Schriften zur Literatur des Mittelalters. Stuttgart 2004, S. 183-196; Albrecht Hausmann: Struktur, Autorisierung, Autorschaft. Untersuchungen zur Poetik von Erec, Gregorius und Tristan. Habilitationsschrift (masch.) Eichstätt 2009; Ludger Lieb: Ein neuer doppelter Kursus in Hartmanns Erec und seine Kontrafaktur in Gottfrieds Tristan. In: DVjs 83 (2009), S. 193-217.

4 Vgl. Michel Huby: L'adaptation courtoise en Allemagne au XII ${ }^{\mathrm{e}}$ et au XIII ${ }^{\mathrm{e}}$ siècle. Paris 1968. Zum Begriff und seiner kontroversen Diskussion in der französisch-deutschen Forschung zuletzt Ricarda Bauschke: Adaptation courtoise als Schreibweise. Rekonstruktion einer Bearbeitungstechnik am Beispiel von Hartmanns Iwein. In: Texttyp und Textproduktion in der deutschen Literatur des Mittelalters. Hrsg. von Elizabeth Andersen, Manfred Eikelmann, Anne Simon. Berlin/New York 2005 (TMP. 7), S. 65-84; Wolfgang Achnitz: Deutschsprachige Artusdichtung des Mittelalters. Eine Einführung. Berlin/Boston 2012, S. $49 \mathrm{f}$.

5 Vgl. Worstbrock (Anm. 3), S. 194 f.; Lieb (Anm. 3); Cornelia Herberichs, Susanne Reichlin (Hrsg.): Kein Zufall. Konzeptionen von Kontingenz in der mittelalterlichen Literatur. Göttingen 2010 (Historische Semantik. 13), darin bes. den einleitenden Beitrag von Susanne Reichlin: Kontingenzkonzep- 
Warning seinerseits trug zur theoretischen Beschreibung dieser Binnenstrukturen bei, indem er Theoreme aus Roman Jakobsons Darstellung der poetischen Funktion der Sprache ${ }^{6}$ auf Erzählstrukturen anwandte: Wie bei der poetischen Funktion der Sprache das Prinzip der Äquivalenz von der Achse der Selektion auf die Achse der Kombination übertragen werde, so übertrügen auch epische Texte Äquivalenzen von der Paradigmatik (der Auswahlmöglichkeit aus Stoffen, Motiven, Figurentypen) auf die Syntagmatik des Erzählzusammenhangs. Diese Form der Binnenwiederholung nennt Warning Erzählen im Paradigma und zeigt auf, dass sie im modernen Roman zur Exposition von Kontingenz genutzt wird. ${ }^{7}$ Doch auch in den mittelalterlichen Erzählungen vermochte Warning dieses Prinzip nachzuweisen, so u. a. an den wiederholten Listhandlungen in Gottfrieds Tristan ${ }^{8}$ und zuletzt an den Inzestwiederholungen in Hartmanns Gregorius, die ihrerseits, mit veränderter Akzentuierung, in Thomas Manns Erwähltem wiedererzählt und in der ironisierenden Selbstermächtigung des Erzählers einer potenziellen Unendlichkeit zugeführt werden. ${ }^{9}$

Wenn sich im Folgenden der solchermaßen für das Phänomen der Wiederholung geschärfte Blick auf Dingwiederholungen konzentriert, ist zu berücksichtigen, dass Dinge zumeist in einer als metonymisch beschreibbaren Beziehung stehen, die sie mit erzählten Handlungen und Figuren, mit Erzählmotiven oder mitunter auch mit dem Erzählen selbst verbindet. Ein berühmtes Beispiel ist die Madeleine in Marcel Prousts Romanzyklus A la Recherche du temps perdu, jenes Gebäck, das der Erzähler in Tee taucht und dabei an seine Kindheit, genauer an die Ferienaufenthalte in dem Provinzstädtchen Combray erinnert wird. ${ }^{10}$ Der Geschmack des aufgeweichten Biskuits evoziert die einstmals in Combray genossene Madeleine, aber auch andere Dinge in deren Umgebung, etwa den Kirchturm von Combray oder den blühenden Weißdornbusch. Gérard Genette hat die Beziehung dieser Dinge als metonymisch beschrieben und deutlich gemacht, dass diese als erinnerte Gegen-

tionen in der mittelalterlichen Literatur. Methodische Vorüberlegungen, S. 11-49, v. a. S. 31-34, 46 f., sowie die Abhandlungen von Albrecht Hausmann: Gott als Funktion erzählter Kontingenz. Zum Phänomen der ,Wiederholung، in Hartmanns von Aue Gregorius, S. 79-109, und Elke Koch: Erzählen vom Tod. Überlegungen zur Finalität in mittelalterlichen Georgsdichtungen, S. 110-130.

6 Vgl. Roman Jakobson: Closing Statement: Linguistics and Poetics. In: Style in Language. Hrsg. von Thomas A. Sebeok. Cambridge, Mass. 1960, S. 350-377; deutsche Übersetzung von Heinz Blumensath und Rudolf Kloepfer: Linguistik und Poetik. In: Literaturwissenschaft und Linguistik. Ergebnisse und Perspektiven. Hrsg. von Jens Ihwe. Bd. II/1: Zur linguistischen Basis der Literaturwissenschaft I. Frankfurt a. Main 1971 (Ars poetica. Texte. 8), S. 142-178.

7 Vgl. Warning, Erzählen im Paradigma (Anm. 1), zu Jakobson bes. S. 178-180.

8 Vgl. Warning, Die narrative Lust (Anm. 1), bes. S. 181-184.

9 Vgl. Rainer Warning: Berufungserzählung und Erzählerberufung. Hartmanns Gregorius und Thomas Manns Der Erwählte. In: DVjs 85 (2011), S. 283-334.

10 Vgl. Marcel Proust: A la Recherche du temps perdu. Vol. 1: Du côté de chez Swann. A l'ombre des jeunes filles en fleurs. Ed. publiée sous la dir. de Jean-Yves Tadié avec, pour ce volume, la collaboration de Florence Callu et al. Paris 1987, S. 44-47. 
stände in Bezug zueinander sowie in Verbindung zu Personen und erzählten Motiven der Welt von Combray stehen, etwa zu der bettlägerigen Tante Léonie, zu dem abendlichen Besucher Charles Swann oder zu dem anlässlich dieser Besuche von der Mutter vorenthaltenen Gutenachtkuss (beim Zubettgehen des erinnerten, damals kindlichen Ich). ${ }^{11}$ Genette stellt diesen metonymischen Beziehungen auf der Ebene des Erinnerten eine metaphorische Beziehung (der Ähnlichkeit) zwischen dem Erinnern und dem Erinnertem der verlorenen Zeit gegenüber. Diese metaphorische Beziehung wird durch den Genuss der Madeleine ausgelöst, welche erst die kindliche Welt von Combray evoziert. Als Erinnerte aber stehen die Dinge und Begebenheiten von Combray in einem metonymischen Zusammenhang (der Nachbarschaft bzw. Berührung). ${ }^{12}$

Im Folgenden sollen Dingwiederholungen in Wolframs Parzival unter dem in den vorgängigen Ausführungen eingenommenen Blickwinkel betrachtet werden. Dabei ist vorab ein Ding zu betrachten, das im Text auch explizit als solches bezeichnet wird: der Gral. Dieser steht mit anderen Dingen wie der blutenden Lanze, den silbernen Messern, dem Gralschwert, aber auch mit Personen wie der Gralträgerin Repanse de Schoye, dem leidenden Anfortas und dem tumben Parzival sowie dem Handlungsgang in metonymischer Nachbarschaft. Durch ausdrücklich in Szene gesetzte Wiederholungsvorgänge wird dabei ein Erzählen im Paradigma betrieben, das eine eigenwillige Ambivalenz zwischen Kontingenzbewältigung und Kontingenzexposition aufweist. Es ist ein Erzähler am Werk, der mit Dingen wie dem Gral oder dem Gralschwert Kohärenzen einerseits stiftet, diese aber andererseits auch destabilisiert. Dabei bleibt mitunter eigenwillig offen, ob der Autor an seiner Stoffbewältigung wenn nicht scheitert, so sich doch an ihr abmüht, oder aber ob er eben dieses Mühen als solches inszeniert. ${ }^{13}$ Das derart ausgestellte Erzählen ist

11 Gérard Genette: Métonymie chez Proust. In: ders.: Figures III. Paris 1972 (Poétique), S. 41-63.

12 Vgl. ebd., S. 63: „c'est la métaphore qui retrouve le Temps perdu, mais c'est la métonymie qui le ranime, et le remet en marche“.

13 Vgl. zum Erzählverfahren in Wolframs Parzival stellvertretend Michael Curschmann: Das Abenteuer des Erzählens. Über den Erzähler in Wolframs Parzival. In: DVjs 45 (1971), S. 627-677; Ulrike Draesner: Wege durch erzählte Welten. Intertextuelle Verweise als Mittel der Bedeutungskonstitution in Wolframs Parzival. Frankfurt a. Main u. a. 1993 (Mikrokosmos. 36); Cornelia Schu: Vom erzählten Abenteuer zum Abenteuer des Erzählens. Überlegungen zur Romanhaftigkeit von Wolframs Parzival. Frankfurt a. Main u. a. 2000 (Kultur, Wissenschaft, Literatur. 2); Fritz P. Knapp: Subjektivität des Erzählers und Fiktionalität der Erzählung bei Wolfram von Eschenbach und anderen Autoren des 12. und 13. Jahrhunderts. In: Wolfram von Eschenbach - Bilanzen und Perspektiven. Eichstätter Kolloquium 2000. Hrsg. von Wolfgang Haubrichs, Eckart C. Lutz, Klaus Ridder. Berlin 2002 (Wolfram-Studien. 17), S. 10-29; Joachim Bumke: Wolfram von Eschenbach. 8. Aufl. Stuttgart/Weimar 2004 (Sammlung Metzler. 36), S. 215-218, mit weiterer Literatur. Gegenüber den komplexen Erzählsituationen in Wolframs Parzival eher blass bleiben die knappen Ausführungen bei Rainer Warning: Narrative Hybriden. Mittelalterliches Erzählen im Spannungsfeld von Mythos und Kerygma (Der arme Heinrich/Parzival). In: Präsenz des Mythos. Konfigurationen einer Denkform in Mittelalter und Früher Neuzeit. Hrsg. von Udo Friedrich, Bruno Quast. Berlin/New York 2004 (TMP. 2), S. 19-33, hier S. 25-33. 
dabei stets auch auf vorhandene oder nur behauptete Vorlagen verwiesen, namentlich auf Chrétiens de Troyes Roman de Perceval ou le Conte du Graal und den geheimnisvollen Gewährsmann Kyot. ${ }^{14}$ Damit ist das Phänomen des authentischen bzw. fiktional inszenierten Wiedererzählens Teil dieser an erzählte Dinge wie etwa den Gral geknüpften Stoffbewältigung.

\section{II ein dinc der Gral}

Die Untersuchung soll mit einer an Überlieferungsbefunde geknüpften Textbeobachtung eingeleitet werden: In Dreißiger 454 aus Buch IX des Parzival findet sich der berühmte Abschnitt, in dem der Gewährsmann eines Gewährsmanns den Gralnamen in den Sternen erblickt (vgl. die Edition im Anhang). ${ }^{15}$ Der Heide Flegetanis, dessen Aufzeichnungen Kyot, wie es kurz davor heißt, in Toledo verworfen ligen vant (V. 453,12), sah den geheimnisvoll verborgenen Gralnamen mit seinen Augen (V. 454,17/19). ${ }^{16}$ Diese Vision aber geht simultan mit einem scheuen Sprechen einher, bei dem Flegetanis den in den Sternen gelesenen Gralnamen auch ausspricht: Das beschreibt der in das phraseologische Syntagma (er) sach [...] mit sînen ougen ${ }^{17}$ eingebundene Nebensatz dâ von er blûclîche sprach (V. 454,18). Was Flegetanis bei diesem Sprechakt sagt, wird folgendermaßen zum Ausdruck gebracht: er jach, ez hiez ein dinc der Grâl (V. 454,21).

14 Vgl. Chrétien de Troyes: Le Roman de Perceval ou Le Conte du Graal. Edition critique d'après tous les manuscrits par Keith Busby. Tübingen 1993 (im Folgenden zitiert). Zu Chrétien und Kyot vgl. die Überblicksdarstellungen bei Bumke (Anm. 13), S. 237-239 (dazu ausführliche Vergleiche mit Chrétien in den Handlungsanalysen S. 61-111) und S. 244-247 („Das Kyotproblem“), jeweils mit weiterer Literatur; zu Kyot auch Michael Stolz: Kyot und Kundrie. Expertenwissen in Wolframs Parzival. In: Wissen, maßgeschneidert. Experten und Expertenkulturen im Europa der Vormoderne. Hrsg. von Björn Reich, Frank Rexroth, Matthias Roick. München 2012 (HZ Beihefte NF. 57), S. 83-113.

15 Alle anderen Zitate aus Wolframs Parzival folgen der Ausgabe: Wolfram von Eschenbach: Parzival. Studienausgabe. Mittelhochdeutscher Text nach der sechsten Ausgabe von Karl Lachmann. Übersetzung von Peter Knecht. Mit Einführungen zum Text der Lachmannschen Ausgabe und in Probleme der Parzival-Interpretation von Bernd Schirok. 2. Aufl. Berlin/New York 2003.

16 Vgl. zur Stelle auch Stolz (Anm. 14), S. 94-96, 100-102; ders.: Von der Überlieferungsgeschichte zur Textgenese. Spuren des Entstehungsprozesses von Wolframs Parzival in den Handschriften. In: Grundlagen. Forschungen, Editionen und Materialien zur deutschen Literatur und Sprache des Mittelalters und der Frühen Neuzeit. Hrsg. von Rudolf Bentzinger, Ulrich-Dieter Oppitz, Jürgen Wolf. Stuttgart 2013 (ZfdA Beihefte. 18), S. 37-61, hier S. $47 \mathrm{f}$.

17 Dieses begegnet beispielsweise auch am Beginn der zweiten (nach Handschrift BC) bzw. dritten (nach Handschrift A) Strophe des Reichstons Walthers von der Vogelweide: Ich sach mit mînen ougen; zit. nach Walther von der Vogelweide: Leich, Lieder, Sangsprüche. 15., veränderte und um Fassungseditionen erweiterte Auflage der Ausgabe Karl Lachmanns. Aufgrund der 14., von Christoph Cormeau bearbeiteten Ausgabe neu hrsg., mit Erschließungshilfen und textkritischen Kommentaren versehen von Thomas Bein. Edition der Melodien von Horst Brunner. Berlin/Boston 2013, S. 13. 
So lautet zumindest die Textversion von Handschrift D (St. Gallen, Stiftsbibliothek, Cod. 857, 2. Drittel des 13. Jahrhunderts), welche dem edierten Text zugrunde liegt. ${ }^{18}$ In der angefügten Musteredition ${ }^{19}$ werden neben dem konstituierten Text die Varianten der übrigen Textfassungen in verkleinertem Schriftgrad und normalisierter Form mit der jeweiligen Sigle angegeben. ${ }^{20}$ In einer ersten Apparatetage sind die berücksichtigten Textzeugen verzeichnet. Eine zweite Apparatetage dokumentiert Gliederungsmittel der erwähnten Handschriften (wie Initialen, Überschriften Illustrationen). In der dritten Apparatetage sind die Abweichungen der Leithandschrift D vom konstituierten Text (z. B. das fehlende Wort heiden in V. 454,1) zusammengestellt. Ferner werden hier (nur von den in der ersten Apparatetage angegebenen Textzeugen) die aussagerelevanten Binnenvarianten der Fassungstexte ${ }^{\star} m{ }^{\star} G{ }^{\star} T$ aufgeführt. Wenn sich die Fassungsvarianten nur in einem Teil der den Fassungen zugeordneten Textzeugen finden, werden diese einzeln angegeben; zudem werden fassungsinterne Varianten dokumentiert, wenn einzelne Textzeugen vom konstituierten Text oder den daneben angeführten Fassungsvarianten abweichen. Wie ein Blick in die Musteredition zeigt, weisen die Fassungen ${ }^{\star} \mathrm{G}$ und ${ }^{\star} \mathrm{T}$ in Vers 454,21 eine kleine, jedoch nicht unbedeutende Variante auf: Das Verbum hiez ist durch wære ersetzt. Dieser Befund ist zunächst unter überlieferungsgeschichtlichen Aspekten bemerkenswert, denn der im neunten Buch befindliche Dreißiger 454 gehört jenem, die Bücher VIII bis XI umfassenden Bereich an, in dem der Gegensatz der Hauptfassungen ${ }^{\star} \mathrm{D}$ und ${ }^{\star} \mathrm{G}$ nahezu inexistent ist. ${ }^{21}$ Der Lesart wære aber folgen hier mit der Ausnahme eines stark kontaminierten Manuskripts

18 Vgl. zu den Handschriftensiglen die Übersichten bei Robert Schöller: Die Fassung *T des Parzival Wolframs von Eschenbach. Untersuchungen zur Überlieferung und zum Textprofil. Berlin/New York 2009 (Quellen und Forschungen zur Literatur- und Kulturgeschichte NF. 56 [290]), S. 57-59; und Klaus Klein: Beschreibendes Verzeichnis der Handschriften (Wolfram und Wolfram-Fortsetzer). In: Wolfram von Eschenbach. Ein Handbuch. Hrsg. von Joachim Heinzle. 2 Bde. Berlin/Boston 2011, Bd. 2, S. 941-1002, hier S. 943.

19 Vgl. zu den Editionsprinzipien des Parzival-Projekts, in dem eine Neuausgabe des Textes vorbereitet wird, Michael Stolz: Chrétiens Roman de Perceval ou le Conte du Graal und Wolframs Parzi$v a l$ - ihre Überlieferung und textkritische Erschließung. In: Wolframs Parzival-Roman im europäischen Kontext. Hrsg. von Klaus Ridder, Susanne Köbele, Eckart Conrad Lutz. Berlin 2014 (WolframStudien. 23), S. 431-478, hier S. 465-471; zu der vorliegenden Musteredition bes. ders.: Von den Fassungen zur Eintextedition. Eine neue Leseausgabe von Wolframs Parzival. In: Überlieferungsgeschichte transdisziplinär. Neue Perspektiven auf ein germanistisches Forschungsparadigma. In Verbindung mit Horst Brunner und Freimut Löser hrsg. von Dorothea Klein. Wiesbaden 2016 (Wissensliteratur im Mittelalter. 52), S. 353-388, vgl. bes. die „Erläuterungen zur Einrichtung des Lesetextes“, S. $387 \mathrm{f}$.

$20 \mathrm{Zu}$ den vier Textfassungen, die alle noch aus dem 13. ( ${ }^{\star} \mathrm{m}$ evtl. aus dem frühen 14.) Jh. stammen und von denen sich jeweils ${ }^{\star} \mathrm{D}$ und ${ }^{\star} \mathrm{m}$ bzw. ${ }^{\star} \mathrm{G}$ und ${ }^{\star} \mathrm{T}$ näher stehen, ausführlich Stolz, Chrétiens Roman de Perceval (Anm. 19), S. 457-459.

21 Dazu Bernd Schirok: Parzival III.1. Die Handschriften und die Entwicklung des Textes. In: Wolfram von Eschenbach. Ein Handbuch (Anm. 18), Bd. 1, S. 308-334, hier S. 320 f.; Stolz (Anm. 16), S. 46, mit weiterer Literatur. 
(Hs. Z) sämtliche zu Fassung ${ }^{\star} G$ gehörenden Handschriften sowie alle die Fassung ${ }^{\star} \mathrm{T}$ konstituierenden Textzeugen. Insgesamt handelt es sich um 11 von insgesamt 16 diesen Vers überliefernde Manuskripte, die in ihrer Gesamtheit den markanten Gegensatz der Fassungen ${ }^{\star} \mathrm{D}$ und ${ }^{\star} \mathrm{m}$ einerseits, ${ }^{\star} \mathrm{G}$ und ${ }^{\star} \mathrm{T}$ andererseits dokumentieren.

Worin aber besteht der Unterschied der beiden Textversionen von Vers 454,21? Beide Male wird mit dem von jach abhängigen Nebensatz die Aussage des Flegetanis in indirekter Rede festgehalten: Gemäß dem Text der Fassungen ${ }^{\star} \mathrm{D}$ (repräsentiert durch Handschrift D) und *m sagt Flegetanis, dass ein dinc der Grâl hieß. Hier erfolgt also im Sprechakt des heidnischen Gewährsmanns die Benennung des Grals. Anders verfährt die Textversion der Fassungen ${ }^{\star} \mathrm{G}$ und ${ }^{\star} \mathrm{T}$ : Dort geht es in den Worten des Flegetanis nicht um den Namen, sondern um die Existenz des Grals: er jach, ez wære ein dinc, der Grâl. Die Aussage, dass das Ding in der einen Version Gral heißt $\left({ }^{\star} \mathrm{D}{ }^{\star} \mathrm{m}\right)$, in der anderen der Gral ist $\left({ }^{\star} \mathrm{G}{ }^{\star} \mathrm{T}\right)$, könnte beinahe an Positionen des hochmittelalterlichen Universalienstreits angebunden werden. Dort ging es bekanntlich um die Frage, ob den Dingen ihre Namen bzw. Begriffe real, d. h. wesenhaft, oder nur nominell, d. h. durch Konvention, zukommen. ${ }^{22}$ Aber die Variante auf diese Dimension hin zu öffnen, würde wohl zu weit führen.

Wichtig ist vielmehr, dass der divergent überlieferte Vers nahezu wörtlich eine Stelle aus dem fünften Buch aufgreift, die im Kontext der von Parzival unverständig beobachteten Gralzeremonie steht. Dort heißt es von dem geheimnisvollen Gegenstand, den Repanse de Schoye auf einem grüenen achmardî (V. 235,20), wertvollem Seidenstoff aus Arabien, ${ }^{23}$ trägt: daz was ein dinc, daz hiez der Grâl (V. 235,23). In diesen Worten klingt der in indirekter Rede stehende Sprechakt des Flegetanis nahezu wörtlich an, diesmal jedoch im Modus einer direkten Aussage seitens des Erzählers: Der kurz zuvor als wunsch von pardîs („paradiesische Vollkommenheit“; V. 235,21) bezeichnete Gegenstand war ein Ding, das der Gral hieß. Der Erzähler gewährt der Imagination seiner Zuhörer damit einen gleichsam unverstellten Blick auf den Gral, der gleichwohl an die Vermitteltheit dichterischer Rede gebunden ist. Die Suggestion der dem Publikum ermöglichten Teilhabe an der Gralzeremonie wird in den umgebenden Versen durch eine Häufung von Imperativen verstärkt, welche an die sinnliche Wahrnehmung appellieren, etwa seht (V. 233,12) oder hœrt (V. 232,12; 234,30; 238,2).

Im Blick auf den in Buch V enthaltenen Vers können auch die Textversionen des Korrespondenzverses in Buch IX nochmals genauer beschrieben werden. Die

22 Vgl. stellvertretend Alain de Libera: La querelle des universaux. De Platon à la fin du Moyen Age. Paris 1996 (Des Travaux), bes. S. 136-141; deutsche Übersetzung von Konrad Honsel: Der Universalienstreit. Von Platon bis zum Ende des Mittelalters. München 2005, S. 142-148.

23 Dazu ausführlicher Michael Stolz: „A thing called the Grail“. Oriental Spolia in Wolfram’s Parzival and its manuscript tradition. In: The Power of Things and the Flow of Cultural Transformations. Art and Culture between Europe and Asia. Hrsg. von Lieselotte E. Saurma-Jeltsch, Anja Eisenbeiß. München/Berlin 2010, S. 188-216, hier S. 189 f. 
Version der Fassungen ${ }^{\star} \mathrm{D}$ und ${ }^{\star} \mathrm{m}$ (er jach, ez hiez ein dinc der Grâl) rückt das Verbum des im Vers von Buch V enthaltenen Relativsatzes (ein dinc, daz hiez der Grâl) in die als indirekte Rede wiedergegebene Aussage des Flegetanis. In direkter Rede wiedergegeben, würde diese lauten: ein dinc hiez der Grâl. Demgegenüber steht die Version der Fassungen ${ }^{\star} \mathrm{G}$ und ${ }^{\star} \mathrm{T}$ (er jach, ez wære ein dinc, der Grâl) der Erzählerrede von Buch V näher. Hier weist die in indirekter Rede formulierte Aussage des Flegetanis eine Ellipse gegenüber dem die Gralzeremonie beschreibenden Vers auf: Es entfällt das aus Relativpronomen und Verbum bestehende Syntagma daz hiez. In direkte Rede übertragen würden die Worte des Flegetanis lauten: $e z$ was ein dinc, der Grâl - nämlich - so wäre sinngemäß zu ergänzen - ein dinc, das der Grâl hieß.

In beiden Textversionen liegt dabei gegenüber dem aus der Beschreibung der Gralzeremonie stammenden Vers ein Prinzip der Äquivalenz vor, das hier - im Sinne Jakobsons - von der paradigmatischen auf die syntagmatische Achse des Erzählablaufs übertragen ist. Es kommt zu einer Wiederholungsstruktur, die sich im Sinne Warnings als Erzählen im Paradigma fassen lässt. Überträgt man diesen auf einer synchronen Ebene angesiedelten Befund in eine diachrone, werkgenetische Dimension, so lässt sich vermuten, dass die beiden Verse mit ihrer auffälligen klanglichen Ähnlichkeit vom Autor korrespondierend zueinander angelegt worden sein dürften. Womöglich rechnete der Autor damit, dass sich sein Publikum anlässlich der Ausführungen zu Flegetanis im neunten Buch der Formulierung des fünften Buchs bewusst oder unbewusst erinnerte. ${ }^{24}$ Dann wäre ein Rezeptionsvorgang angezielt, der ähnlich wie die von Proust beschriebene unwillkürliche Erinnerung (mémoire involontaire) einen quasi metaphorischen Bezug herstellt: Das von Flegetanis benannte Ding der Gral gemahnt an die den Hörern bereits bekannte Gralzeremonie mit all den ihr zugehörigen Komponenten wie Gralträgerin, Lanze, silbernen Messern und Gralschwert. Die hierbei assoziativ hergestellten Beziehungen zu den Dingen und Personen wären - in Genettes Terminologie - metonymischer Natur. Vielleicht ist der für den Rezeptionsvorgang wenn nicht notwendig intendierte, so doch naheliegende Wiedererkennungseffekt auch der Grund dafür, dass sich die

24 In diese Richtung zielt auch, ausgehend von einer grundlegenden Studie zur Zeitstruktur, die Argumentation von Hermann J. Weigand: Die epischen Zeitverhältnisse in den Graldichtungen Crestiens und Wolframs. In: Publications of the Modern Language Association 53 (1938), S. 917950; englische Übersetzung vom Verf.: Narrative Time in the Grail Poems of Chrétien de Troyes and Wolfram von Eschenbach. In: ders.: Wolfram's Parzival. Five essays with an Introduction. Hrsg. von Ursula Hoffmann. Ithaca/London 1969, S. 18-74, hier S. 73: „Wolfram ingeniously inserts some necessary data as early as Book V [...] he indulges his pleasure in a game of hide and seek by teasing the reader's curiosity regarding the revelations to come.“ Ders.: A Jester at the Grail Castle in Wolfram's Parzival. In: Publications of the Modern Language Association 67 (1952), S. 485-510, Nachdruck in ders.: Wolfram's Parzival. Five essays, S. 75-119, sieht die Bezüge zwischen Buch V und IX kompositorisch ,as an organic whole“ angelegt (S. 94), muss dabei aber selbst die „possibility of revision, of changes and interpolations“ (S. 95) seitens des Autors einräumen. Dazu ausführlicher unten, S. 276, 288 f. mit Anm. 85. 
zwischen den beiden Textversionen bestehende Variante von hiez vs. wære gehalten hat, obwohl die Unterschiede der Hauptfassungen ${ }^{\star} \mathrm{D}$ und ${ }^{\star} \mathrm{G}$ in diesem Bereich sonst nahezu verschwinden. Zu erwähnen bleibt schließlich, dass in der Abfolge der beiden weit auseinander liegenden Verse Erzählzeit und erzählte Zeit, Discoursund Histoire-Ebene gegenläufig angelegt sind:25 Der Erzähler macht bei der ersten Erwähnung des Dings Gral anlässlich der Gralzeremonie im fünften Buch eine Aussage, die zeitlich nach der Vision des Flegetanis und dem darin eingebundenen Sprechakt liegt. Der von Flegetanis ausgesprochene Gralname und dessen Niederschrift in heidenischer schrifte (V. 453,13) bzw. in latînschen buochen (V. 455,4) sind ja Voraussetzungen dafür, dass der Erzähler über die Vermittlung des Kyot den Gral nun seinerseits beim Namen nennen kann. Der auf der Discours-Ebene erst später, im neunten Buch, erfolgende Visionsbericht geht damit auf der HistoireEbene voran.

Diese gegenläufige Bewegung ist Teil jener Erzählstrategie des Parzival-Romans, bei der dem Publikum Informationen vorbehalten und thematische Zusammenhänge erst allmählich aufgedeckt werden. ${ }^{26}$ Der Rezipientenschaft wird auf diese Weise die Perspektive Parzivals aufgezwungen, der die Geheimnisse des Grals - verholenbæriu tougen, wie es im Kontext der Flegetanis-Vision heißt (V. 454,20) - seinerseits erst nach und nach durchschaut. Eine besonders konsequente Korrelation besteht dabei gerade zwischen dem fünften Buch, das Parzivals ersten Besuch auf der Gralburg und die unterlassene Mitleidsfrage beinhaltet, und dem neunten Buch, in dem Parzival durch Trevrizent über die Gralgeheimnisse aufgeklärt wird.

In einem einschlägigen Aufsatz, der Wolframs Parzival „als Lesemysterium“ deutet, hat Harald Haferland gezeigt, dass die stets aufgeschobene Suche nach den Geheimnissen des Grals ein konstantes Thema in den Conte du Graal-Dichtungen sowohl auf der Histoire- als auch auf der Discours-Ebene darstellt, dies besonders in Texten, die sich als Fortsetzungen an Chrétiens de Troyes Conte du Graal anlagern: „Man darf von dem oder den Gralgeheimnis(sen) nicht sprechen, solange die Erzählung nicht selbst bis zu diesem Punkt gelangt ist.“27

25 Die Begriffe Histoire und Discours folgen Tzvetan Todorov: Les catégories du récit littéraire. In: Communications 8 (1966), S. 125-151, bes. S. 126, übernommen von Gérard Genette: Discours du récit. In: ders.: Figures III (Anm. 11), S. 65-273, hier S. 71 f., als „histoire“ und „récit“.

26 Zum Verfahren prägnant Bumke (Anm. 13), S. 210-215, hier v. a. S. 215: „Zu begreifen, wie das Nicht-Zusammenhängende zusammenhängt und das Nicht-Zusammenpassende zusammenpasst, scheint das Ziel des Erkenntniswegs zu sein, auf den der Parzival-Erzähler seine Zuhörer (und seine späteren Interpreten) geschickt hat.“

27 Harald Haferland: Die Geheimnisse des Grals. Wolframs Parzival als Lesemysterium? In: ZfdPh 113 (1994), S. 23-51, hier S. 47. Vgl. zu den Conte du Graal-Fortsetzungen die Überblicksdarstellungen bei Albert Wilder Thompson: Additions to Chrétien's Perceval - Prologues and Continuations. In: Arthurian Literature in the Middle Ages. A Collaborative History. Hrsg. von Roger Sherman Loomis. Oxford 1959, S. 206-219; Matilda Tomaryn Bruckner: Chrétien Continued. A Study of the Conte du Graal and its Verse Continuations. Oxford 2009, S. 4 f.; Thomas Hinton: The Conte du Graal- 
So möchte Perceval beispielsweise gemäß der altfranzösischen, wenige Jahre vor Wolframs Parzival entstandenen zweiten Fortsetzung (des Wauchier de Denain, Ende des 12. Jahrhunderts) vor seinem zweiten Besuch auf der Gralburg wissen, was für ein Ding (chose, der Terminus begegnet also auch hier) der Graal sei, den er vormals in der Gralburg gesehen hatte: Quel chose li Graaux estoit, / Que il leanz veü avoit (V. $32061 \mathrm{f}$.). Von dem wertvollen und kostbaren Gral (Li riches Graaux precieux; V. 32064) heißt es, dass niemand über ihn sprechen solle, wenn er dazu nicht die Befugnis - umschrieben als alle Güter oder Gnaden der Welt - habe (Que nus hom parler n'an devroit / Se toz les biens dou mont n'avoit; V. 32065 f.). ${ }^{28}$

In der (bald?) nach der zweiten Fortsetzung abgefassten Elucidation, einer prologartigen, in ihren Aussagen dunkel bleibenden Erläuterung der Gralthematik, ${ }^{29}$ wird betont, dass niemand vor der rechten Zeit über die Gralsgeheimnisse berichten dürfe (nus ne doit / Le secret dire ne chonter; / [...] ains qu'il fust tos dis; V. 47). ${ }^{30}$ Mehrfach wird dabei „auf erst später zu Erzählendes“ verwiesen, ${ }^{31}$ ähnlich wie dies im fünften Buch des Parzival im Kontext des Bogengleichnisses geschieht, wenn es von dem greisen Titurel heißt: Wer der selbe wære, / des freischet her nâch mære (V. 241,1f.). ${ }^{32}$

Mit der Erwähnung der Flegetanis-Vision im neunten Buch ist eben jener Moment erreicht, an dem es der Erzähler unternimmt, die Geheimnisse des Grals im

Cycle. Chrétien de Troyes' Perceval, the Continuations, and French Arthurian Romance. Cambridge 2012 (Gallica. 23), S. 244 (Appendix 2, Inhaltsangaben ebd., S. 229-243, Appendix 1); Leah Tether: The Continuations of Chrétien's Perceval. Content and Construction, Extension and Ending. Cambridge 2012 (Arthurian studies. 79), S. 1; sowie Stolz, Chrétiens Roman de Perceval (Anm. 19), S. 437440, mit weiterer Literatur.

28 Zit. nach: William Roach (Hrsg.): The Continuations of the Old French Perceval of Chretien de Troyes. 4 Bde. Philadelphia 1949-1971, Bd. 4, S. $492 \mathrm{f}$.

29 Sie geht in der Handschrift Mons, Bibliothèque de l'Université de Mons-Hainaut, 331/206 (4. Viertel des 13. Jh., picardisch) Chrétiens Perceval ou le Conte du Graal und dessen Fortsetzungen voraus. Ausgabe der Handschrift (textkritisch unzureichend): Chrestien de Troyes: Perceval le Gallois. Publié d'après le manuscrit de Mons par Charles Potvin. 6 Bde. (Publications de la Société de Bibliophiles Belges, séant à Mons. 21). Mons 1865-1871. Dazu die Angaben in der Conte du GraalAusgabe von Busby (Anm. 14), S. XXIII-XXV; und bei Terry Nixon: Catalogue of Manuscripts. In: Les Manuscrits de Chrétien de Troyes. The Manuscripts of Chrétien de Troyes. Hrsg. von Keith Busby u. a. 2 Bde. Amsterdam 1993 (Faux titre. 71/72), Bd. 2, S. 1-85, hier S. 54-56; Stolz, Chrétiens Roman de Perceval (Anm. 19), bes. S. 440, mit weiterer Literatur.

30 Albert Wilder Thompson (Hrsg.): The Elucidation. A Prologue to the Conte del Graal. New York 1931, S. 86. Zur Datierung Thompson (Anm. 27), S. 207-209, hier S. 208: „The only clue to the date of composition of the Elucidation is the fact that its author knew the first two continuations."

31 Haferland (Anm. 27), S. 47, Anm. 88.

32 Vgl. zum Bogengleichnis die Ausführungen bei Weigand, Narrative Time (Anm. 24), S. 70-72, und Bumke (Anm. 13), S. 205 f., 212, mit weiterer Literatur. Erwägungen im Hinblick auf die Fassungsproblematik (Varianten in Fassung ${ }^{\star} \mathrm{T}$ ) bei Schöller (Anm. 18), S. 284-294; und im Hinblick auf mögliche Entstehungsbedingungen ( ${ }^{\star} \mathrm{T}$ als die mutmaßlich älteste Fassung) bei Michael Dallapiazza, Alessandra Molinari: Wolframs Parzival und das Problem des festen Textes. Die Varianten des Bogengleichnisses. In: Filologia germanica 3 (2011), S. 47-70. 
Verweis auf die Mittlerinstanzen Flegetanis und Kyot sowie in den erzähllogisch daran anschließenden Ausführungen Trevrizents zu lüften. In gewisser Weise ist dafür Ausgangspunkt jener Sprechakt, in dem Flegetanis das Ding mit dem Namen Gral benennt, als er die Sternenschrift entziffert. Der mit Scheu ausgesprochene Name ermöglicht es dem Erzähler, den Namen seinerseits im fünften Buch zu einem Zeitpunkt auszusprechen, als er im Zuge einer Fokalisierung auf Parzivals Wahrnehmung die Gralgeheimnisse noch nicht zu offenbaren vermag. ${ }^{33}$ Die Wiederholung des ähnlich lautenden Verses deutet, zumal mit den Überlieferungsvarianten in den Textfassungen, auf Alternativen eines Verständnisses des Grals als Ding, das ist, bzw. als Ding, das heißt, indem es mit einem bestimmten Namen benannt wird. Zugleich weist die Wiederholung auf Phänomene des Wiedererzählens in der Conte du Graal-Tradition hin: Die Strategie des Vorenthaltens von Informationen zu den Gralgeheimnissen findet sich auch in den französischen Anlagerungen an Chrétiens Perceval-Roman. Es ist nicht gänzlich auszuschließen, dass Wolfram von Texten wie der Elucidation oder der zweiten Fortsetzung Kenntnis hatte, was von deren Entstehungszeit her wohl möglich wäre. Wolfram hätte in diesem Fall rezentes Material der französischen Tradition zur Verfügung gehabt und bearbeitet. ${ }^{34}$ Auf methodisch sichererem Boden bewegt sich freilich die Annahme, dass die erwähnten Fortschreibungen der Conte du Graal-Tradition und Wolframs Parzival unter vergleichbaren, auf der Basis von Chrétiens Ausgangstext beruhenden Voraussetzungen, zu ähnlichen Erzählstrategien gelangten.

Ausgehend von diesen Überlegungen soll nun im zweiten Teil des Beitrags die metonymische Nachbarschaft des Dings mit dem Namen Gral betrachtet werden.

\section{Das Gralschwert und seine metonymischen Kontexte}

In den Blick geraten dabei jene Gegenstände, die Parzival sieht, als er beim ersten Besuch auf der Gralburg die geheimnisvolle Gralzeremonie (mit Prozession und

33 Vgl. zum Begriff der Fokalisierung („focalisation“) Genette (Anm. 25), S. 214 f.: „C’est en général le ,point de vue du héros‘ qui commande le récit, avec ses restrictions de champ, ses ignorances momentanées.“ Dazu im Hinblick auf die höfische Epik ausführlich Gert Hübner: Erzählform im höfischen Roman. Studien zur Fokalisierung im Eneas, im Iwein und im Tristan. Tübingen/Basel 2003 (Bibliotheca Germanica. 44).

34 Vgl. zum umstrittenen Grad von Wolframs Kenntnissen der französischen Sprache und Literatur stellvertretend Eberhard Nellmann: Zu Wolframs Bildung und zum Literaturkonzept des Parzival. In: Poetica 28 (1996), S. 327-344, und Weigand, A Jester (Anm. 24), S. 87-90; zusammenfassend Bumke (Anm. 13), S. 8 f., und Heiko Hartmann: Darstellungsmittel und Darstellungsformen in den erzählenden Werken. In: Wolfram von Eschenbach. Ein Handbuch (Anm. 18), Bd. 1, S. 145-220, hier S. 147-149. 
Speisewunder) verfolgt. Bei Wolfram sind das die blutende Lanze, diverse Leuchter sowie Tisch- und Geschirrvorrichtungen, die silbernen Messer, der Gral und das Gralschwert (V. 231,15-240,12). ${ }^{35}$ Letzteres erhält Parzival von Anfortas zum Abschluss der Gralzeremonie als Geschenk; an die Gabe knüpft sich, wie einem Klageruf des Erzählers (ôwê daz er niht vrâgte dô!; V. 240,3) zu entnehmen ist, die von Parzival erwartete Frage.

In Chrétiens Conte du Graal (V. 3190-3319) sind die Komponenten der Gralzeremonie, der auch dort etwas Geheimnisvolles anhaftet, anders geordnet: Hier steht die Schwertgabe am Beginn, wobei das Schwert hier von der Nichte des Fischerkönigs stammt, was bei Wolfram nicht erwähnt wird. Anschließend erfolgt die Prozession mit der blutenden Lanze, goldenen Leuchtern, dem graal, silbernen Tellern und diversen Tischvorrichtungen für die Mahlzeit. Neben der veränderten Ordnung wären nun Unterschiede in zahlreichen Erzähldetails erwähnenswert und ausführlich zu erläutern, was jedoch im Folgenden aus Platzgründen und im Hinblick auf die spezifische Frage nach den Dingwiederholungen unterbleiben soll. Nur en passant seien die allgemein bekannten Tatsachen erwähnt, dass der Gral bei Chrétien als eine mit Edelsteinen verzierte Goldschale, bei Wolfram als wertvoller Edelstein beschrieben wird, dass bei Chrétien von einem Mahl zu zweit, bei Wolfram von einem allgemeinen Festmahl mit Speisewunder die Rede ist, dass bei Chrétien von Perceval erwartet wird, dass er nach der Funktion der Lanze und des Grals fragt, während er bei Wolfram nach dem Leiden des Gralkönigs fragen soll. ${ }^{36}$ Wolframs silberne Messer, die auch im neunten Buch nochmals eine wichtige Rolle spielen, dürften zudem auf einem Missverständnis beruhen: Ihnen entspricht bei Chrétien ein silberner Teller (tailleoir d'argant; V. 3231) - Wolfram oder ein ihn beratender Vermittler hat das altfranzösische Wort tailleoir wohl von taillier, „schneiden“, abgeleitet. $^{37}$

Anstelle dieser Besonderheiten soll im Folgenden das Gralschwert näher betrachtet werden, welches sowohl bei Chrétien als auch bei Wolfram ein rätselhafter Gegenstand ist, dessen änigmatischer Charakter an den Gralgeheimnissen teilhat, ohne je ganz aufgelöst zu werden. ${ }^{38}$ Möglicherweise stellt das Schwert ein unbe-

35 Vgl. die tabellarische Übersicht in: Wolfram von Eschenbach: Parzival. Nach der Ausgabe Karl Lachmanns revidiert und kommentiert von Eberhard Nellmann. Übertragen von Dieter Kühn. 2 Bde., Frankfurt a. Main 1994 (Bibliothek des Mittelalters. 8,1/2), Kommentarteil, Bd. 2, S. 574; zum Erzählverfahren Johannes Maczewski: Wolframs Erzähltechnik im ersten Munsalvaesche-Abschnitt des Parzival. In: Seminar 20 (1984), S. 1-26.

36 Vgl. zusammenfassend den Kommentar von Nellmann (Anm. 35), S. 574-585; und Bumke (Anm. 13), S. 70 f., 135-139.

37 Vgl. den Kommentar von Nellmann (Anm. 35), S. 578 f.; ders.: Produktive Missverständnisse. Wolfram als Übersetzer Chrétiens. In: Übersetzen im Mittelalter. Cambridger Kolloquium 1994. Hrsg. von Joachim Heinzle, L. Peter Johnson, Gisela Vollmann-Profe. Berlin 1996 (Wolfram-Studien. 14), S. 134-148, hier S. 140-142.

38 Vgl. dazu Weigand, A Jester (Anm. 24), S. 92 f. mit Anm. 22; Walter Mersmann: Der Besitzwechsel und seine Bedeutung in den Dichtungen Wolframs von Eschenbach und Gottfrieds von Straß- 
wältigtes Relikt aus einer älteren Tradition dar. ${ }^{39}$ Aber gemäß dem zuvor eingeschlagenen Weg, Texte miteinander zu vergleichen, ohne schon von vornherein diachrone Zusammenhänge zu postulieren, erscheint es problematisch, hier auf nicht mehr rekonstruierbare Ursprünge zu rekurrieren. Unabhängig von solchen Überlegungen ist das Gralschwert ein Musterbeispiel für Dingwiederholungen in dem eingangs beschriebenen Sinne, denn es begegnet bei Chrétien und Wolfram intratextuell an verschiedenen Stellen und es lässt in einer intertextuellen Perspektive erkennen, dass an dem Motiv erzählend weitergearbeitet wurde, so in diversen Interpolationen und Fortsetzungen von Chrétiens Conte du Graal. ${ }^{40}$

Bei Chrétien wird das Schwert von einem Knappen (vallés; V. 3131 u. ö.) vor den Fischerkönig gebracht, woraufhin dieser es feierlich an Perceval übergibt, welcher im Roman, so auch anlässlich der Gralzeremonie, seinerseits als Knappe bzw. junger Mann (vallet; V. 3166 u. ö.) bezeichnet wird (V. 3131-3186). Diese begriffliche Überschneidung sowie eine Häufung von im Hinblick auf das Subjekt unscharf bestimmter Verben im Kontext der Übergabe ${ }^{41}$ tragen ihrerseits dazu bei, die metonymischen personellen Bezüge, in welchen das Schwert steht, $\mathrm{zu}$ verschleiern. Soviel aber scheint klar zu sein, dass das Schwert eine Aufschrift mit Angaben zu seiner Herkunft trägt: Car en l'espee estoit escrit (V. 3137). Das hat er gesehen, wie es im altfranzösischen Text heißt: $i$ vit (V. 3138; ähnlich: Si vit; V. 3136). Die wahrnehmende Person - vermutlich der Fischerkönig - sieht offenbar bei Lektüre der Inschrift, dass das aus Edelstahl gefertigte Schwert nicht zerbrechen kann, außer in einer großen Gefahr - par i. tot seul peril -, von der nur der Hersteller weiß (V. 3141-3143).

Wolfram bringt die Schwertgabe wie erwähnt am Schluss der Gralzeremonie, ohne an dieser Stelle eine Inschrift zu erwähnen. Damit entfällt auch der Hinweis auf das Risiko, dass das Schwert zerbricht. Der deutsche Text enthält jedoch eine ergänzende - wie sich zeigen wird problematische - Angabe mit dem Hinweis des Anfortas, dass er dieses Schwert vor seiner Verletzung im Kampf oft mit sich geführt habe: ,hêrre, ich prâhtz in nôt / in maneger stat ${ }^{\star}$ (V. 239,25 f.). Nach der

burg. München 1971 (Medium Aevum. 22), S. 135-137; Werner Schröder: Parzivals Schwerter. In: ZfdA 100 (1971), S. 111-132; Petrus W. Tax: Nochmals zu Parzivals zwei Schwertern. Ein nachdenklicher und narrativ-kombinatorischer Versuch über Schwerter und Kampfstrategien, Segen und Impotenzen in Wolframs Parzival. In: ZfdA 135 (2006), S. 275-308; Friedrich E. Grünzweig: Das Schwert bei den Germanen. Kulturgeschichtliche Studien zu seinem Wesen vom Altertum bis ins Hochmittelalter. Wien 2009 (Philologica Germanica. 30), S. 299-304, 403; sowie den Kommentar von Nellmann (Anm. 35), S. $584 \mathrm{f}$.

39 So Schröder (Anm. 38), S. 117.

40 Dazu Stolz, Chrétiens Roman de Perceval (Anm. 19), S. 437 f., 472 f.; und unten, S. 287 f.

41 Vgl. z. B.: Si vit bien ou ele fu faite (V. 3136), i vit / Qu'ele estoit de si bon achier (V. 3138 f.), Subjekt ist wohl jeweils der Fischerkönig, Objekt (ele) das Gralschwert (espee); Celui qui ses armes gardoit / I vit, et si li comanda / L'espee, et cil la li garda (V. 3182-3184), Subjekt ist Perceval, der das Schwert nunmehr seinem Knappen übergibt. 
Schwertgabe ist Parzival jedenfalls im Besitz zweier Waffen: des Gralschwerts und jenes Schwerts, das er bereits zuvor dem toten Ither geraubt hat. ${ }^{42}$

Näheren Aufschluss über das Schwert erhält der Protagonist sowohl bei Chrétien als auch bei Wolfram erst anlässlich der Begegnung mit seiner Cousine, die im deutschen Text den Namen Sigune trägt. Angesichts ihres Verwandtschaftsverhältnisses zu Perceval/Parzival wäre es sogar möglich, dass es sich bei der Cousine um die Nichte des Fischerkönigs handelt, von der nach Chrétien - nicht aber nach Wolfram - das Schwert stammt. ${ }^{43}$ Jedenfalls weiß die Cousine erstaunlich gut über das Schwert Bescheid: Sie kennt den Hersteller sogar mit seinem Namen Triboët (V. 3679). ${ }^{44}$ Sie erwähnt, dass das Schwert - anders als dann bei Wolfram - noch nie in Gebrauch war. Und sie warnt Parzival davor, sich auf die Waffe zu verlassen, denn sie werde im Kampf in Stücke brechen: en grant bataille, / [...] ele vos volera en pieces (V. 3662f.). Eine Reparatur des Schwerts könne nur der Schmied Triboët leisten, der am See von Cothoatre wohne (Au lac qui est soz Cothoatre; V. 3675). ${ }^{45}$ Weiter hat Chrétien das Motiv des Gralschwerts in seinem Roman nicht mehr behandelt.

Wolfram hingegen gestaltet es zu einem Netzwerk von Dingwiederholungen aus, das sich nahezu über die gesamte Dichtung des Parzival erstreckt. In der an den Besuch auf der Gralburg Munsalvæsche anschließenden Sigune-Szene - bei Wolfram der zweiten Begegnung mit der Cousine - spielt das Schwert eine zentrale Rolle. Bereits unmittelbar davor erwägt Parzival, als er den Spuren der Gralritter folgt, dass er das Schwert unverdient mit sich führe (ungedient ich daz trage; V. 248,29).

42 Vgl. die Schilderung der Szene am Morgen nach der Gralprozession und der verpassten Mitleidsfrage: Ufem teppech sach der degen wert / ligen sîn harnasch und zwei swert: / daz eine der wirt im geben hiez, / daz ander was von Gaheviez (V. 246,1-4), zwei swert er umbe gurte (V. 246,26). Bei Gaheviez handelt es sich um die Heimat Ithers (vgl. V. 145,15f.: ez was Ithêr von Gaheviez: / den rôten riter man in hiez).

43 So sagt der Knappe zum Fischerkönig in Bezug auf das herbeigebrachte Gralschwert: ,Sire, la sore pucele, / Vostre niece qui tant est bele, / Vos a envoié cest present“ (V. 3145-3147).

44 Nach anderen Handschriften Trabuchet, Trebucet u. ä.; vgl. das Variantenverzeichnis zur Stelle in: Kristian von Troyes: Der Percevalroman (Li contes del Graal). Unter Benutzung des von Gottfried Baist nachgelassenen handschriftlichen Materials hrsg. von Alfons Hilka. Halle a. d. Saale, S. 1932 (Christian von Troyes. Sämtliche erhaltene Werke. 5), S. 166.

45 Damit dürfte Scottewatre, der alte Name für den schottischen, in die Nordsee mündenden Fluss und anschließenden Meeresarm Firth of Forth (Ästuar nördlich von Edinburgh) gemeint sein. Die Präposition soz würde nahelegen, dass es sich bei Cothoatre um eine Burg handelt (so auch in der Fortsetzung des Gerbert de Montreuil); die in einer Vielzahl von Handschriften überlieferte Präposition sur deutet hingegen auf einen Wasserlauf hin. Vgl. den Kommentar in der Ausgabe von Busby (Anm. 14), S. 476. Zur Deutung von Cothoatre als Scottewatre auch Alexander Bell: Zu Perceval 3675: Cotöatre. In: ZfrPh 54 (1934), S. 753-755; Chrétien de Troyes: Le Roman de Perceval ou le Conte du Graal. Publié d'après le ms. fr. 12576 de la Bibliothèque Nationale par William Roach. Genève/Lille 1956. $2^{\mathrm{e}}$ édition revue et augmentée Genève/Paris 1959 (Textes Littéraires Français. 71), S. 282; G. D. West: An Index of Proper Names in French Arthurian Verse Romances 1150-1300. Toronto 1969 (University of Toronto Romance Series. 15), S. 46 a. 
Bei Sigune angekommen, erhält Parzival von seiner Verwandten Auskünfte über die Gralfamilie und eben auch über das Schwert (Dreißiger 253 und 254, vgl. wiederum die Edition im Anhang). Sigune erwähnt das von dem Gralkönig Anfortas stammende Schwert erstmals in Vers 253,24 (dû vüerest ouch umbe dich sîn swert) und knüpft daran sofort den Hinweis, dass es im Kampf genutzt werden könne, sofern Parzival den Segen des Schwerts kenne (V. 253,25 f.). Von Segensworten spricht Sigune auch später nochmals (V. 254,15). Zuvor aber erläutert sie die Eigenart des Schwerts: Es wurde von dem Schmied Trebuchet hergestellt (V. 253,28f.). Als dessen Aufenthaltsort nennt Wolfram - im Gegensatz zu dem bei Chrétien erwähnten See von Cothoatre - die Quelle von Karnant, also die Heimat Erecs (V. 253,30 f.). Er erlaubt sich den Witz, den Namen von Erecs Vater, der gemäß Hartmann von Aue „Lac“ heißt (Êrec fil de roi Lac; V. 2 u. ö.), ${ }^{46}$ von dem entsprechenden französischen Wort für See abzuleiten, das Chrétien an dieser Stelle (V. 3675) verwendet. ${ }^{47}$ In Vers 254,8 wird der Name Lac dann auch für die Quelle beansprucht (der selbe brunne heizet Lac; so gemäß den Fassungen ${ }^{\star} \mathrm{m}{ }^{\star} \mathrm{G}{ }^{\star} \mathrm{T}$, in ${ }^{\star} \mathrm{D}$ fehlt das Wort brunne). Im Kontext dieser Namensnennungen gibt Sigune eine Gebrauchsanweisung für den Fall, dass das Schwert zerbricht. Dabei spielt das Quellwasser von Lac eine wichtige Rolle, denn es erlaubt zum rechten Zeitpunkt, vor Sonnenaufgang, die Zusammenfügung des Schwerts (V. 254,4-14). Dabei würden, so Sigune, auch valz und ecke - die Vertiefung an der Längsseite des Schwerts und der Schneidenrand - wieder hergestellt (V. 254,12f.). Und diu mâl, womit wohl die auch bei Chrétien (V. 3137) erwähnte Inschrift gemeint ist, ${ }^{48}$ würden ihren Glanz (schîn) und damit ihre Lesbarkeit nicht verlieren: und vliesent niht diu mâl ir schîn (V. 254,14).49 In diesem Zusammenhang kommt Sigune nun in Vers 254,15 erneut auf die Segensworte zu sprechen, deren das Schwert zu seiner Wiederherstellung bedarf (daz swert bedarf wol segens wort) - es handelt sich dabei wohl um einen auf dem Schwert eingravierten Segen. 50

46 Vgl. Erec von Hartmann von Aue. Mit einem Abdruck der neuen Wolfenbütteler und Zwettler Erec-Fragmente. Hrsg. von Albert Leitzmann, fortgeführt von Ludwig Wolff. 7. Auflage besorgt von Kurt Gärtner. Tübingen 2006 (ATB. 39), S. 1.

47 Vgl. den Kommentar von Nellmann (Anm. 35), S. 593; zur Stelle auch Stolz (Anm. 16), S. 45 f.

48 Vgl. Schröder (Anm. 38), S. 125, 130.

49 In dem zu Fassung *m gehörenden Fragment 69 aus der ersten Hälfte des 14. Jh. fehlt die Verneinung nicht, was ermöglichen würde, für diese Fassung eine die Verse 254,14f. umgreifende Syntax anzunehmen: „wenn die Inschrift ihren Glanz verliert, bedarf das Schwert der Segensworte“. $\mathrm{Zu}$ diesem Textzeugen ausführlich Thomas Franz Schneider, Gabriel Viehhauser: Zwei Neufunde zu Wolframs von Eschenbach ,Parzival‘. Teil 2: Das dreispaltige Solothurner Fragment F 69. Ein Vertreter der ,Nebenfassung ${ }^{\star}{ }^{\star} \mathrm{m}$. In: Reflexion und Inszenierung von Rationalität in der mittelalterlichen Literatur. Blaubeurer Kolloquium 2006. Hrsg. von Klaus Ridder, Wolfgang Haubrichs, Eckart Conrad Lutz. Berlin 2008 (Wolfram-Studien. 20), S. 457-525 und Abb. 5-40.

50 Die Gebrauchsanweisung wird dabei mit einem zweifach negierten Konditionalsatz zum Ausdruck gebracht (Sint diu stucke niht verrêrt / [...] und vliesent niht diu mâl ir schîn; „wenn die Stücke nicht verstreut sind [...] und die Inschrift nicht ihren Glanz verliert“; V. 254,9/14). 
Dass das Gralschwert tatsächlich einen Segensspruch trägt, deutet eine auf Trebuchet bezogene Äußerung Trevrizents im neunten Buch an: den list tet im [Trebuchet] ein segen kuont, / der an des küneges swerte stuont (V. 490,23f.). Allerdings bezieht sich dieser Segen auf die Herstellung der silbernen Messer durch Trebuchet und nicht auf die Restitution des Gralschwerts. Im Hinblick auf den auf dem Schwert eingravierten Segensspruch (diu mâl; V. 254,14) ist davon auszugehen, dass Parzival diesen nicht lesen kann, denn es gibt keinerlei Anzeichen dafür, dass er vor seinem ersten Besuch in Munsalvæsche lesen gelernt hat, weder in Soltane noch bei Gornemanz noch bei anderer Gelegenheit. ${ }^{51}$ Damit kann Parzival die Segensworte nicht entziffern und Sigune, die zumindest gemäß den Angaben in Wolframs Titurel-Bruchstücken lesen können sollte, ${ }^{52}$ wird wohl nach den Regeln der Gralgemeinschaft nicht bereit sein, Parzival den Spruch vorzulesen. Dass derjenige, der den Spruch kennt, das Zerbrechen des Schwerts im Kampf erst gar nicht $\mathrm{zu}$ befürchten braucht - darauf deuten Sigunes Ausführungen in Vers 253,25 f.: bekennestû des swertes segen, / dû maht ân angest strîtes pflegen. ${ }^{53}$

Wie Vers 254,16 belegt, ahnt Sigune jedoch, dass Parzival die Segensworte auf Schloss Munsalvæsche gelassen hat: ich vürhte, diu hâstû lâzen dort. ${ }^{54}$ Dieses lâzen kann als „Unterlassen“ verstanden werden; dann würden die Segensworte eng an die verpasste Mitleidsfrage angebunden, ja mit dieser identifiziert werden. ${ }^{55}$ Es kann aber auch als „dort lassen“, im Sinne von „nicht erfahren“ aufgefasst werden. ${ }^{56}$ Auf diese Vereindeutigung zielt die in Fassung ${ }^{\star} \mathrm{T}$ (und einigen ${ }^{\star} \mathrm{m}$ - und ${ }^{\star} \mathrm{G}$ Textzeugen wie Fragment 69 und Handschrift L) belegte Form gelâzen. In diesem

51 Vgl. auch Tax (Anm. 38), S. 287 f. - Parzivals Analphabetismus ist seinerseits Thema in der modernen Version des Romans von Adolf Muschg, wo Parzival während der Einkehr beim Einsiedler Trevrizent anhand einer Fibel mit alliterativen Einträgen zu jedem einzelnen Buchstaben lesen und damit auch seine Identität zu begreifen lernt. Vgl. Adolf Muschg: Der Rote Ritter. Eine Geschichte von Parzivâl. 6. Aufl. Frankfurt a. Main 1995, S. 648-660.

52 Im Hinblick auf das verlorene Brackenhalsband verlangt Sigune nâch der schrift an dem seile (Strophe 168,4). Vgl. Wolfram von Eschenbach: Titurel. Hrsg., übersetzt und mit einem Kommentar und Materialien versehen von Helmut Brackert und Stephan Fuchs-Jolie. Berlin 2002, S. 152; bzw. Wolfram von Eschenbach: Titurel. Mit der gesamten Parallelüberlieferung des ,Jüngeren Titurel‘. Kritisch hrsg., übersetzt und kommentiert von Joachim Bumke und Joachim Heinzle. Tübingen 2006, S. 435 (als Strophe 157,4, gemäß der an Handschrift G, München, Bayerische Staatsbibliothek, Cgm 19, orientierten Zählung).

53 So der Text gemäß den Fassungen ${ }^{\star} D{ }^{\star} m$; in den Fassungen ${ }^{\star} G{ }^{\star} T$ beginnt das Verspaar mit den Worten: hâstû gelernt [...]. Die Interpretation, dass die Kenntnis des Segens das Zerbrechen des Schwerts verhindert, auch bei Weigand, A Jester (Anm. 24), S. 93, Anm. 22, und im Kommentar von Nellmann (Anm. 35), S. 593.

54 Woher Sigune ihr Wissen über die Geschehnisse auf Munsalvæsche am Vortrag bezieht, bleibt unklar. Vgl. zur Problematik beispielsweise Weigand, A Jester (Anm. 24), S. 111-113 (,it is possible to assume [...] that she saw in her mind's eye the scene enacted on the night before“; S. 113) und den Kommentar von Nellmann (Anm. 35), S. 594 f.

55 So Schröder (Anm. 38), S. 125.

56 So der Kommentar von Nellmann (Anm. 35), S. 594. 
Fall wären die auf Munsalvæsche gelassenen Segensworte weniger eng mit der versäumten Mitleidsfrage verbunden. Sie wären dann allenfalls eine Folge des Versäumnisses.

Wenn die auf dem Schwert eingravierten Segensworte auf Munsalvæsche bekannt sind, dürften sie auch Anfortas vertraut sein. In diesem Fall (und nur in diesem) hätte es eine Logik, wenn Anfortas sagt, er habe das Gralschwert im Kampf benutzt (hêrre, ich prâhtz in nôt / in maneger stat; V. 239,25f.). Auch eine spätere Stelle, in der es heißt, Trebuchet habe Frimutels Schwert (mit einem Segen?) graviert, wäre dann auf das Gralschwert beziehbar (Trebuchet der smit, / der Frimutels swert ergruop, / dâ von sich starkez wunder huop; V. 643,18-20). Es würde deutlich, dass dieses vom Großvater Frimutel über den Oheim Anfortas auf Parzival übergeht. ${ }^{57}$ Allerdings hat es den Anschein, dass Wolfram der Kampfkraft des Schwerts nicht recht traut. Auffällig ist auch der in der Überlieferung unterschiedlich gestaltete Versuch, die Dauer jener Phase, ehe das Schwert zerbricht, auszudehnen: Gemäß der Mehrzahl der Fassungen tritt dieser Moment nicht beim ersten, sondern erst beim zweiten Schlag ein: daz swert gestêt ganz einen slac, / am andern ez zevellet gar (V. 254,2 f.). Laut Fassung *T währt die aufrechterhaltene Intaktheit gar einen tac (V. 254,2). 58

Im Anschluss an die in Vers 254,16 geäußerte Befürchtung, dass Parzival den Segen auf Munsalvæsche verpasst haben könnte, kommt Sigune in Vers 254,17 auf die alternative Möglichkeit zu sprechen, dass Parzival - genauer: sein muot bzw. sein munt (so die Fassungen ${ }^{\star} \mathrm{m}{ }^{\star}{ }^{\star} \mathrm{G}{ }^{\star} \mathrm{T}$ ) - die Segensworte gelernet haben könnte. Damit leitet sie nun auf die heilbringenden Folgen der Mitleidsfrage über, vorausgesetzt, dass diese gestellt worden wäre. Auf diese Weise stehen die auf dem Schwert eingravierten Segensworte und die Mitleidsfrage erneut in einem engen Zusammenhang. Nicht nur den Schwertsegen, sondern alles Glück auf Erden hätte Parzival erworben, wenn er nur die Frage gestellt hätte: niemen ist sô rîche, / der gein dir koste mege hân, / hâstû vrâge ir reht getân (V. 254,28-30).

Auch an anderen Stellen erfolgt diese Rückbindung des Gralschwerts an die unterlassene Mitleidsfrage, so, wie bereits erwähnt, wenn der Erzähler kurz nach der Schwertübergabe beklagt, dass Parzival die Frage nicht gestellt habe (V. 240,3), 59 ja suggeriert, dass die Schwertgabe geradezu eine Ermahnung zur Frage gewesen sei (wan do erz enpfienc in sîne hant, / dô was er vrâgens mit ermant;

57 Vgl. ebd., S. 738 f.; anders Schröder (Anm. 38), S. 129 f.

58 Dazu Tax (Anm. 38), S. 279 f.; Schöller (Anm. 18), S. 363 f. Tax versucht den in den meisten Fassungen begegnenden Wortlaut zu retten, indem er dem Gralschwert eine „Erstschlagkapazität“ (Tax [Anm. 38], S. 279 u. ö.) zuschreibt, die den Kampf mit einem einzigen Schlag entscheiden würde. In Fassung *T wäre diese Erstschlagkapazität „einem Eintagesschwert gewichen“ (Schöller [Anm. 18], S. 364). - Die Variante könnte freilich auch auf eine Augenabirrung ([...] ê in beschîne der tac; V. 254,7) zurückgehen.

59 Vgl. oben, S. 277. 
V. 240,5f.). ${ }^{60}$ Ähnlich ist auch der von Verfluchungen begleitete Tadel, den Parzival wegen der unterlassenen Frage durch Sigune und später durch Kundrie erfährt, thematisch mit dem Gralschwert verbunden. ${ }^{61}$ Dabei werden, jeweils repetitiv, der Anblick des Grals, ferner die zur Prozession gehörenden silbernen Messer und die blutende Lanze (snîdnde silbr und bluotec sper) ${ }^{62}$ sowie die unterlassene Frage erwähnt. Hier zeigt sich also auch eine Rekurrenz der mit dem Gral metonymisch verbundenen Dinge. Zugleich lässt sich eine Rückkoppelung an Chrétiens Text erkennen, denn dort wird von Perceval - anders als bei Wolfram - nicht erwartet, dass er sich nach dem Befinden des Gralkönigs erkundigt, sondern dass er nach der Eigenart der blutenden Lanze, des silbernen Tellers und des Grals fragt. Eben diese Versäumnisse erwähnt der französische Text ausdrücklich. ${ }^{63}$

Die nächsten, das Gralschwert betreffenden Dingwiederholungen finden sich erst im neunten Buch, das in den vorausgehenden Ausführungen zur Erleichterung des Textverständnisses bereits verschiedentlich herangezogen worden ist. Das Verfahren, dunkle Stellen aus einem Abschnitt der Dichtung durch solche aus einem anderen, weit entfernten Abschnitt zu erläutern, ist dabei methodisch nicht ganz unproblematisch, da es impliziert, dass durch die Beziehbarkeit solcher Passagen Kohärenz gestiftet werden kann. ${ }^{64}$ Die bislang hergestellten und im Folgenden noch herzustellenden Bezüge erfolgen dementsprechend mit Vorbehalt und dem Eingeständnis, dass es vielleicht angemessener wäre, die Widersprüchlichkeit der Aussagen als solche offenzulegen.

Erwähnt wird das Gralschwert im neunten Buch an prominenter Stelle, nämlich im Anschluss an das Gespräch mit der personifizierten frou âventiure, das den Erzähler auf Parzivals Spuren zurücklenkt. ${ }^{65}$ In der Folge ist mehrfach davon die Rede, dass die âventiure uns, also wohl dem Erzähler und seinem impliziten Publikum, bekant gibt (V. 434,11) bzw. kündet (V. 435,2), was Parzival erlebt habe. Dabei bleibt offen, ob hier noch die personifizierte frou âventiure spricht oder von der

60 Später erklärt Trevrizent freilich, dass es wirkungslos gewesen wäre, wenn Parzival eine ihm nahegelegte Frage gestellt hätte: V. 483,24-28.

61 In der Sigune-Szene schließt der entsprechende Abschnitt (V. 255,1-20) unmittelbar an die Ausführungen zum Gralschwert an. Bei der Verfluchung durch Kundrie (V. 316,21-317,2) wird der Abschnitt eingeleitet durch das Verspaar: iu gap iedoch der wirt ein swert, / des iwer wirde wart nie wert; V. 316,21 f.

62 Identisch im Wortlaut von V. 255,11 u. 316,27.

63 Si s'est de demander tenus (V. 3204 in Bezug auf die in V. 3196-3201 erwähnte blutende Lanze: Le lance blanche et le fer blanc - S'issoit une goute de sanc). - Ne n'osa mie demander/ Del graal cui l'en en servoit (V. 3244 f.; nachdem kurz davor der tailleoir d'argant und li graals, qui aloit devant erwähnt worden sind; V. 3231 f.). - Ne li vallés ne demanda / Del graal cui on en servoit (V. 3292f.). Vgl. zur blutenden Lanze auch unten, S. 286 f. mit Anm. 78, und S. 289, Anm. 85.

64 So verfährt im Prinzip Weigand (Anm. 24), vgl. die dort angeführten Stellen.

65 Stellvertretend für die Fülle von Forschungsliteratur sei auf Bumke (Anm. 13), S. 207, und die in Anm. 13 genannten Arbeiten verwiesen. 
âventiure als Erzählung bzw. Quelle die Rede ist. In diesem Zusammenhang erfolgt in den Versen 434,25-30 ein kurzer Hinweis auf das Gralschwert:
sîn swert, daz im Anfortas
gap dô er bîme grâle was,
brast sît dô er bestanden wart:
dô machtez ganz des brunnen art
bî Karnant, der dâ heizet Lac.
das swert gehalf im prîss bejac.

Das von Anfortas verliehene Schwert soll also tatsächlich zerbrochen sein, als Parzival angegriffen wurde. Doch die Zauberkraft der Quelle Lac bei Karnant habe es, wie von Sigune angekündigt, wiederhergestellt. Das Schwert habe Parzival zu Ruhm (prîs) verholfen. Mit der launigen Bemerkung Swerz niht geloubt, der sündet (V. 435,1) leitet der Erzähler sodann zu Parzivals dritter Begegnung mit Sigune über. Es hat den Anschein, als solle das sperrige, aus dem fünften Buch übrig gebliebene Motiv vom Gralschwert abgehandelt werden, ehe die nächste SiguneSzene eröffnet wird. ${ }^{66}$

Die Möglichkeit, das Schwert in einem der vorausgehenden oder folgenden Zweikämpfe, die Parzival ausficht, zum Einsatz zu bringen, hat der Autor nicht genutzt. ${ }^{67}$ Erst spät, anlässlich von Parzivals Zweikampf mit Feirefiz, wird dann tatsächlich das Zerbrechen eines Schwerts beschrieben, doch handelt es sich dabei um Ithers Waffe: von Gaheviez daz starke swert / mit slage ûfs heidens helme brast (V. 744,10 f.). Das Versagen der Waffe führt dazu, dass der Kampf endet und das gegenseitige Morden der Brüder verhindert wird. ${ }^{68}$

Ausführlichere Erwähnung findet das Gralschwert zuvor in der Rede Trevrizents (Dreißiger 501, vgl. wiederum die Edition im Anhang). Am Ende des Karfreitags, an dem er Parzival die Gralgeheimnisse offenbart, kommt der Einsiedler auf das Schwert zu sprechen. ${ }^{69}$ Dabei lehnt sich der Wortlaut des Eingangsverses Din

66 Möglicherweise handelt es sich textgenetisch um einen späteren Einschub; vgl. Stolz (Anm. 16), S. 44-47, mit weiterer Literatur, und unten, S. 288 f., Anm. 85.

67 Eine Liste der Möglichkeiten bei Schröder (Anm. 38), S. 129.

68 Vgl. zu Gaheviez, der Heimat Ithers, oben, Anm. 42; zur Stelle den Kommentar von Nellmann (Anm. 35), S. 760.

69 Die Zeitangabe Karfreitag erschließt sich aus der Rede des grauen Ritters, dem Parzival vor der Einkehr bei Trevrizent begegnet: ,ez ist hiute der karfrîtac‘ (V. 448,7). Darauf, dass Trevrizents Rede in Dreißiger 501 am Ende des Karfreitags stattfindet, deuten die nachfolgenden Zeitangaben sus was er dâ vünfzehen tage (V. 501,11) und Eines tages vrâgt in Parzival (V. 501,19) hin. Allerdings weist Fassung ${ }^{\star} \mathrm{T}$ in Vers 501,19 die Variante Aber sprach dô P. auf, was damit zusammenhängt, dass die mit einer Zeitangabe verbundene inquit-Formel hier bereits in Vers 500,23 erfolgt: Eines tages vrâget in $P$. (gegenüber dô sprach aber $P$. in ${ }^{\star} \mathrm{D}{ }^{\star} \mathrm{G}$ bzw. Aber sprach $P$. in ${ }^{\star} \mathrm{m}$ ). Trevrizents Ausführungen zum Gralschwert sind in Fassung *T also bereits auf einen späteren Zeitpunkt nach dem Karfreitag verschoben. - Zum Zeitgerüst des Romans grundlegend Weigand, Narrative Time (Anm. 24). 
œheim gap dir ouch ein swert (V. 501,1) auffallend eng an einen Korrespondenzvers in der Rede der Sigune an, wo es heißt: dû vüerest ouch umbe dich sîn swert (V. 253,24). Anschließend erfolgt die bereits aus den vorausgehenden Passagen bekannte Anbindung des Schwerts an die versäumte Mitleidsfrage: Parzival habe eine große Sünde begangen, als er die Frage nicht stellte (dâ mit dû sünden bist gewert; V. 501,2). Wie im Kontext des von Sigune erwähnten Schwertsegens (V. 254,17) wird dabei Parzivals munt erwähnt (V. 501,3). Zugleich macht Trevrizent mit der Aussage die sünde lâ bî den andern stên! (V. 501,5) deutlich, dass die unterlassene Frage zu den beiden anderen, zuvor erwähnten Sünden - dem Verwandtenmord an Ither, dem Tod der Mutter Herzeloyde beim Abschied aus Soltâne (V. 499,20-25) - hinzukommt, von diesen möglicherweise sogar übertroffen wird. ${ }^{70}$

Die Szene enthält zugleich eine Verdichtung der eingangs entwickelten Phänotypik von Dingwiederholungen im Parzival, die an das Erzählen im Paradigma, an die metonymischen Beziehungen und an die verzögerte Mitteilung von Informationen geknüpft ist. In dem mit Vers 501,19 beginnenden Abschnitt spricht Parzival seinen Oheim Trevrizent mit einer Frage an und demonstriert damit ausgerechnet gegenüber dem Bruder des Anfortas, dass er es gelernt hat, seinen mütterlichen Verwandten Fragen zu stellen. Die Frage lautet: wer was ein man, lac vorme Grâl? (V. 501,20) ${ }^{71}$ Die in der Frage zum Ausdruck gebrachte lokale Position des Greises ist beispielhaft für die metonymische Beziehung, welche die in der Gralzeremonie erwähnten Dinge mit den Figuren der Dichtung, namentlich den Angehörigen der Gralfamilie, eingehen. Wenn Trevrizent Parzivals Frage in Vers 501,22 dahingehend beantwortet, dass es sich bei dem Greis um Titurel handelt, löst er den vom Erzähler anlässlich des Bogengleichnisses (V. 241,1 f.) angekündigten Aufschub ein. ${ }^{72}$ Die Textstelle ist damit symptomatisch für die Wolframs Erzählen eigene Verzögerung in der Aufdeckung der Gralgeheimnisse. In Handschrift T (Textgrundlage von Fassung *T) erhält diese Verschiebung in der Gewährung von Informationen dadurch eine besondere Akzentuierung, dass Trevrizent in seiner Antwort anstelle des Präteritums (daz was Titurel) das Präsens wählt: Der wirt sprach: ,daz ist Tyturel, / der ist al grâ bî liehtem vel (V. 501,21 f., so der neben dem konstituierten Text stehende Fassungstext). ${ }^{73}$

An der Stiftung solcher Bezüge aber, dies zeigt der Kontext, sind Dinge wie der Gral und das Gralschwert ganz wesentlich beteiligt. In ihrer Wiederholung trans-

\footnotetext{
70 Vgl. stellvertretend zur Diskussion der jeweiligen Versabschnitte in der Forschung den Kommentar von Nellmann (Anm. 35), S. 700-702, sowie Bumke (Anm. 13), S. 87, 91, 126-128.

71 In den für die Fassungen G bzw. T konstitutiven Handschriften GI und T ist vor lac das Relativpronomen der eingefügt.

72 Vgl. zum Bogengleichnis oben, S. 275.

73 Hierbei ist die in den übrigen Handschriften (und damit Fassungen) Parzivals Frage angefügte (und dort im Präteritum ausgedrückte) Charakterisierung des Titurel als der was al grâ bî liehtem vel (dort V. 501,21) aufgrund einer Vertauschung der Verse 501,21/22 der Rede des Trevrizent zugeteilt.
} 
portieren sie wichtige, ihnen metonymisch angelagerte Bestandteile der Erzählung, wie Parzivals Versagen auf der Gralburg und das damit zusammenhängende Nichtverstehen der Geheimnisse der Gralzeremonie. Den Dingen haftet damit menschliche Schwäche, menschliche Krankheit, menschliches Versagen regelrecht an. Selbst die im Erzählvorgang offenbar werdenden Unzulänglichkeiten lassen sich unter dieser metonymischen Relation fassen: Die Schwierigkeiten, die Geheimnisse des Grals einem kohärenten Konzept zu integrieren, werden gerade an Dingen wie dem Gralschwert offenkundig.

Die einem solchen Erzählen eigenen Kontingenzen werden auch auf HistoireEbene zum Ausdruck gebracht, so prominent in Trevrizents spätem Eingeständnis ich louc durch ableitens list / vome grâl, wiez umb in stüende (V. 798,6f.), das sich wohl, wie vielfach betont wurde, auf seine Aussage zu den neutralen Engeln bezieht. ${ }^{74}$ Doch ist Trevrizents Bekenntnis symptomatisch für ein Versagen, zu dem sich bis zu einem gewissen Grad auch der Erzähler und der hinter diesem stehende Autor bekennen. Die handschriftliche Überlieferung bezeugt übrigens Irritationen mit dieser Textstelle und reagiert mit Tilgungen (Hss. L, V') und Zuschreibungen des Satzes an andere Personen wie Feirefiz. ${ }^{75}$

So weit, Dinge wie das Schwert als „Herrschaftszeichen“76 oder als „Dingsymbol verscherzter Königswürde und versäumter Menschlichkeit“ $77 \mathrm{zu}$ interpretieren, wie es die ältere Forschung tat, muss man dabei gar nicht gehen. Es erscheint angemessener, den Dingen im Parzival eine Funktion auf der Ebene des Bezeichnenden, weniger des Bezeichneten zuzusprechen - eines Bezeichnenden, welches in einer repetitiven Paradigmatik das Ingangkommen und die Fortsetzung des Erzählvorgangs auf der syntagmatischen Linie vorantreibt.

\section{Ausblick}

Unter diesem Aspekt wären weitere Dinge der Conte du Graal-Tradition in den Blick zu nehmen. $\mathrm{Zu}$ erinnern wäre an die blutende Lanze aus Chrétiens Perceval und

74 Vgl. dazu die Textstellen V. 471,15-29; 798,11-15, 24-26, sowie Weigand, A Jester (Anm. 24), S. 93 f.; ferner die Kommentare zu V. 798,6f. von Nellmann (Anm. 35), S. 776, Bumke (Anm. 13), S. $119 \mathrm{f}$., und Michaela Schmitz: Der Schluss des Parzival Wolframs von Eschenbach. Kommentar zum 16. Buch. Berlin 2012, S. 97-101.

75 Versausfälle in den Handschriften L (zu Fassung ${ }^{\star} G$ gehörig, Fehlverse 798,1-30) und V' (hier zu Fassung ${ }^{\star} m$ gehörig, Fehlverse 797,13-798,30), Zuschreibungen an Feirefiz in den Handschriften $\mathrm{m}$ n o (zu Fassung ${ }^{\star} \mathrm{m}$ gehörig), an Trevrizent und Parzival in Handschrift R (hier zu Fassung ${ }^{\star} \mathrm{T}$ gehörig).

76 Schröder (Anm. 38), S. 122; danach der Kommentar von Nellmann (Anm. 35), S. 584; vgl. auch Meersmann (Anm. 38), S. 135: „Standes- oder Amtszeichen“.

77 Schröder (Anm. 38), S. 126. 
deren widersprüchliche Gestaltung in Wolframs Text. ${ }^{78} \mathrm{Zu}$ erinnern wäre an den buntgefärbten Speer von Troys, der in der Nähe von Trevrizents Klause herumsteht, als Parzival vor Orilus auf einer Reliquienkapsel einen Eid ablegt und versichert, dass er dessen Frau Jeschute nicht vergewaltigt habe; später spielt dieser Speer in der Blutstropfenszene, Parzivals Herausfallen aus der Zeitlichkeit, und bei der Psalterszene in Trevrizents Klause, Parzivals Zurückfinden in die Zeitlichkeit, eine wichtige Rolle. ${ }^{79} \mathrm{Zu}$ erinnern wäre an den zweimal erwähnten Seidenstoff, der König Artus’ Tafelrunde markiert. ${ }^{80} \mathrm{Zu}$ erinnern wäre schließlich an Interpolationen in Handschriften mit Chrétiens Conte du Graal, wo (nach Vers 3926) das Motiv des zerbrochenen Gralschwerts anlässlich Percevals Kampf mit dem Ritter li Orgueilleus (bei Wolfram: Orilus) weiter ausgestaltet wird. ${ }^{81}$ Dabei gelangt (in den Conte du Graal-Handschriften P und H) ein von der Gralburg ausgesandter Bote auf den Kampfplatz, um die Splitter des im Kampf mit Orgueilleus zerborstenen Gralschwerts wieder aufzusammeln und in die Gralburg zurückzubringen. In den Conte $d u$ Graal-Fortsetzungen wird das Motiv dann mehr oder weniger konsequent in weiteren Erzählzusammenhängen aufgegriffen. ${ }^{82}$ Manche Erzähldetails dieser Interpolationen stimmen dabei mit solchen in Wolframs Parzival überein, auch wenn dieser das Gralschwert im Kampf Parzivals mit Orilus nicht nennt. Als Parallelen wären Jeschutes banges Verfolgen des Kampfes (Wolframs Parzival, V. 262,25-27), der Zorn des Orilus (Wolframs Parzival, V. 264,1-3; vgl. dazu die Interpolation P, V. 44-53), und die Tatsache zu nennen, dass in den Interpolationen im Gegensatz zu Chrétiens vagen Angaben mehrfach von einem Zerbrechen des Schwerts beim ersten Schlag die Rede ist (a cele premiere envaïe, Interpolation P, V. 3; au premier estor, ebd., V. 75; au premier cop, ebd. V. 88 u. 138; ähnlich Interpolation H, V. 30,

78 Vgl. Wolframs Parzival, V. 255,11 (zweite Sigune-Szene in Buch V); 316,27 (Verfluchung durch Kundrie in Buch VI); 489,30-490,2; 492,19-493,8 (Unterredung von Trevrizent und Parzival in Buch IX). In Chrétiens Conte du Graal blutet die Lanze (vgl. Anm. 63), bei Wolfram ist sie gemäß Trevrizents Ausführungen in Buch IX blutig. Dazu Weigand, A Jester (Anm. 24), S. 96-98; Maczewski (Anm. 35), S. 17-19; den Kommentar von Nellmann (Anm. 35), S. 575 f., mit weiterer Literatur; sowie ders. (Anm. 37), S. 142-144, und ders.: Parzival (Buch I-VI) und Wigalois. Zur Frage der Teilveröffentlichung von Wolframs Roman. In: ZfdA 139 (2010), S. 135-152, hier S. 148 f.; ferner unten, S. 288 f., Anm. 85.

79 Vgl. Wolframs Parzival, V. 268,28-30; 271,10-13 (beide Stellen im Kontext der Schwurszene von Buch V); 288,16-20 (Bluttropfenszene in Buch VI); 459,24-460,27 (bes. 460,16-21, Einkehr bei Trevrizent in Buch IX in Zusammenhang mit der Psalterszene, dazu auch Stolz [Anm. 16], S. 48-50). Dies wiederum mit einem wörtlichen Anklang in den Versen 271,12 und 460,20: des vergaz der wilde [460,20: mîn friunt] Taurîân.

80 Vgl. Wolframs Parzival, V. 309,12-22 (Buch VI) und die Dreißiger 775-777 (Buch XV). Dazu die Kommentare von Nellmann (Anm. 35), S. 615, 766, und den Beitrag von Bruno Quast im vorliegenden Band.

81 Dazu ausführlicher Stolz (Anm. 16), S. 41 f., und ders., Chrétiens Roman de Perceval (Anm. 19), S. 472 f., Texte in der Ausgabe von Busby (Anm. 14), S. 395-415.

82 Vgl. Stolz, Chrétiens Roman de Perceval (Anm. 19), S. 437-440. 
124, 207), was mit dem von Sigune in Vers 254,2f. (Buch V) erwähnten andern [slac] (also: dem zweiten Schlag) besser kompatibel wäre. ${ }^{83}$ Da der wie Perceval als valet bezeichnete Bote auf seinem Weg zum Kampfplatz auch bei Percevals Cousine vorbeikommt, wäre in diesem Typus von Interpolation eine mehrfache Sigune-Begegnung, wie sie Wolfram im Gegensatz zu Chrétien gestaltet, zumindest angelegt. ${ }^{84}$ Methodisch sollte man sich bewusst sein, dass es sich bei der Analyse solcher an das Gralschwert gebundenen Bezüge um nicht mehr als Textvergleiche handelt. Wenn man diese prinzipiell synchrone Betrachtung in eine diachrone Linie zu bringen vermöchte, würde sich dabei auch die Frage stellen, welchen Typus von Textvorlagen Wolfram bei seiner Arbeit am Parzival benutzte und ob es dabei womöglich zu Vorlagenwechseln kam. Die Frage, welche Methodik für ein solches Vorgehen angemessen und ob dabei angesichts der Überlieferungssituation der Chrétien-Handschriften je sicheres Terrain zu gewinnen ist, müsste in weiteren Studien geklärt werden. ${ }^{85}$

83 Die erwähnten Textstellen in der Ausgabe von Busby (Anm. 14), S. 396, 398, 400, 406-409. Dazu wiederum ausführlicher Stolz (Anm. 16), S. 42, mit Verweis auf Jean Fourquet: Wolfram d'Eschenbach et le Conte del Graal. Les divergences de la tradition du Conte del Graal de Chrétien et leur importance pour l'explication du texte du Parzival. Paris 1938 (Publications de la faculté des lettres de l'Université de Strasbourg. 87). Überarbeitete Neuauflage. Paris 1966 (Publications de la Faculté des lettres et sciences humaines de Paris-Sorbonne. Série „Etudes et Méthodes“. 17), hier S. $98 \mathrm{f}$., und weitere Literatur.

84 Dazu ausführlicher mit Belegstellen Stolz (Anm. 16), S. 50.

85 Einen Versuch, Möglichkeiten einer textgenetischen Betrachtungsweise aufzuzeigen, bietet der Beitrag von Stolz (Anm. 16). Dort wird in Bezug auf Fourquet (Anm. 83) und die daran anschließenden Forschungen von Nellmann (Anm. 37), bes. S. 145, und ders. (Anm. 78), bes. S. 144-146, ein Weg aufgezeigt, wie ein textgenetischer Ansatz verfahren könnte. Fourquet ging davon aus, dass Wolfram (auf der Grundlage einer der Perceval-Handschrift R nahestehenden Vorlage) zunächst an den Büchern III-VI und vermutlich auch an Teilen von Buch IX gearbeitet habe. In einer zweiten Arbeitsphase habe Wolfram dann eine andere Conte du Graal-Vorlage zur Verfügung gestanden, welche eine Version der ersten Fortsetzung und eine Interpolation (nach V. 3926, wie sie in den Perceval-Handschriften $\mathrm{P}, \mathrm{H}$ fassbar ist) beinhaltet habe; daraus habe Wolfram eine Überarbeitung der Bücher III-VI und die Gawan-Partien des Parzival hergestellt. Denkbar wäre, dass auch eine Überarbeitung von Entwürfen zu Buch IX im Hinblick auf die neue Vorlage erfolgt ist. Folgende Textanteile könnten beispielsweise dieser zweiten Überarbeitungsphase angehören:

- Teile der detaillierten Beschreibung der Fertigung des Gralschwerts durch Trebuchet mitsamt der Erwähnung der Quelle Lac und der damit verbundenen Wiederherstellung in der Rede der Sigune (V. 253,24-254,19, Buch V), vgl. oben, S. 279 f.;

- das mit hoher Wahrscheinlichkeit einen Einschub darstellende Waffenregister des Orilus, in dem eingangs erwähnt wird, dass Trebuchet den Helm des Ritters hergestellt habe (V. 261,1-30, Buch V), dazu mit weiterer Literatur Stolz (Anm. 16), S. 51 mit Anm. 50;

- die ebenfalls als Einschub geltenden Ausführungen zu Bruch und Wiederherstellung des Gralschwerts (V. 434,25-30, Beginn von Buch IX), dazu oben, S. 283 f., und ausführlicher Stolz (Anm. 16), S. 44-47;

- der in Zusammenhang mit Parzivals Zurückfinden in die Zeit in der Unterredung von Parzival und Trevrizent erwähnte Speer von Troys (V. 460,16-23, Buch IX), mit Bezugnahme auf V. 268,28-30; 271,10-13 (Buch V), einschließlich wörtlichen Entsprechungen zwischen den Ver- 
sen 460,20 und 271,12, dazu oben S. 287 mit Anm. 79, und Stolz (Anm. 16), S. 48-50 (mit dem Hinweis, dass die Verse 460,22 f. mit der entscheidenden Zeitangabe in Handschrift T fehlen), skeptisch gegenüber einer Überarbeitung (aber damit nicht durchweg überzeugend) ist Weigand, Narrative Time (Anm. 24), S. 58, Anm. 35, S. 69 f. u. ö.;

- Trevrizents Ausführungen zur in Anfortas Wunde gestoßenen und deshalb blutigen Lanze (daz sper bloutec rôt; V. 490,2, Buch IX), welche im Rahmen der Gralprozession (entsprechend Chrétien) als blutende Lanze beschrieben wird (an der snîden huop sich pluot; V. 231,20), dazu oben, S. 283 mit Anm. 63 und S. 286 f. mit Anm. 78, sowie Nellmann (Anm. 37), S. 142-144, und ders. (Anm. 78), S. $148 \mathrm{f}$;

- Trevrizents Erläuterungen zu den von Trebuchet verfertigten Silbermessern, mit denen die vergiftete Lanzenspitze gereinigt wird (V. 490,13-24, Buch IX), dazu Nellmann (Anm. 37), S. $141 \mathrm{f.,}$ und ders. (Anm. 78), S. 149 (Hinweis auf einen missglückten Überarbeitungsvorgang gegenüber der Funktion der Silbermesser in der Gralzeremonie: zwei messer snîdende; V. 234,18; snîdnde silber und bluotec sper; V. 255,11; jeweils Buch V).

Weiterer Prüfung bedürfte die Frage, ob sich in der für Fassung ${ }^{\star} T$ konstitutiven Handschrift $T$ Textschichten finden, die einen älteren Bearbeitungszustand repräsentieren; darauf könnte beispielsweise der Ausfall der die Zeitangabe zu Parzivals Irrfahrten enthaltenden Verse 460,22f. (s. o.) hindeuten. Eine ausführliche Untersuchung zu textgenetischen Fragen, in die auch die Überlieferungsvarianten von Chrétiens Conte du Graal und dessen Fortsetzungen einbezogen werden, bereitet Dr. Stefan Abel (Bern) vor. 
*D

253 Des ist dîn houbet blôz getân. zem fôrest in Prizlian sah ich dich dô vil minneclîch, swie dû wærest jâmers rîch.

5 dû hâst verlorn varwe und kraft. dîner herten geselleschaft verdrüzze mich, solt ich die haben. wir sulen disen tôten man begraben. Dô nazzeten diu ougen ir die wât.

10 ouch was vroun Lunetten rât ninder dâ bî ir gewesen. diu riet ir vrouwen: "lât genesen disen man, der den iweren sluoc. er mac ergetzen iuch genuoc. $«$

15 Sigune gerte ergetzens niht als wîp, die man bî wanke siht, manege, der ich wil gedagen. hœret mêr Sigunen triwe sagen. diu sprach: »sol mich iht gevröun,

20 daz tuot ein dinc, ob in sîn töun læzet, den vil trûrigen man. schiede dú helflîche dan, sô ist đîn lîp wol prîses wert. dû vüerest ouch umbe dich sîn swert.

25 bekennestû des swertes segen, dû maht ân angest strîtes pflegen.

Sîn ecke ligent im rehte. von edelem geslæhte worht ez Trebuchetes hant. 30 ein brunne stêt bî Karnant. gestân * $G$

in dem f. ze Br. ${ }^{*} G$

vil om. "m

und $\mathrm{om} \cdot \mathrm{*}$

die] si ${ }^{*} G * T$

disen] den ${ }^{*}$ G Fr 69

ir diu oug. ir wât ${ }^{*} G \quad$ ir oug. die wât ${ }^{*} T$ enwas * $T$

diu r.: "vrouwe ${ }^{*} G$

der iu den iw. sl. *G

er mac es erg. i. gen. ${ }^{*} m$ er mac iu sîn erg. gen. ${ }^{*} T$ sine g. ${ }^{*} G$

maneger ${ }^{*} G$ genuoge ${ }^{*} T$, wil ich ${ }^{*} T$

von $\mathrm{S}$. sag. ${ }^{*} \mathrm{G}$ von $\mathrm{S}$, tr. sag. ${ }^{*} T$

si spr. ${ }^{*} G{ }^{*} T$. und sol ${ }^{*} T$

daz ist ${ }^{*} G * T$. in om. ${ }^{*} G$

der vil getriwe man ${ }^{*} G$ den vil getriuwen man ${ }^{*} T(L$ )

lîp] prîs " $m$

hâstu gelernt ${ }^{*} G\left({ }^{*} T\right)$

${ }^{*} D: D-{ }^{*} m$ : $m$ Fr69 (253.8-10, 13-15, 21-22)

*G: GIOLZ - *T: TUVW Fr26 (253.16-27)

1 Initiale $D G \quad 9$ Majuskel $D$ Initiale $m$ Fr69 $O$ (unausg.) $L$ Versal $T \quad 15$ Initiale Z Versal T 19 Initiale I Majuskel T 27 Initiale D W (vorgez.) Fr26 Majuskel T

1 gestan $G I O L \quad 2$ in dem voreis ze (in $L$ ) Br. $G(I L)$ - zů $\mathrm{Br} . W 3$ vil om. $m$ dô] doch $Z$ om. $W \quad 5$ und $o m . T \quad 6$ dines hertzen g. $L \quad 7$ die] si ${ }^{*} G T(U W)$. haben] sehe $U \quad \mathbf{8}$ begrab $Z \quad \mathbf{9}$ ir div ǒgen ir wat $G$ ir ovgen div wât $T$. diu om. $O$. die om. $I U 10$ en was $T(U W) \quad 11$ Mender do $m \quad$. ir om. $W \quad 12$ div riet frơwe $G$ 13 der den iren sl. $m$ der iv den iwern sl. $G(I) \cdot \operatorname{man}]$ wann $W$. den om. $O \quad \mathbf{1 4 . 1 5}$ da von ir herze iamer trúc / er mach ergezzen evch $\sin$ nih $I$, î̂ $\sin$ erg. gn. $T(U V)$ sein eúch erg. gn. $W$. sine gerte $G$, S. gerte ergetzendes n. $V$ S. vergessens gerte n. $W$ 16 jez wip $Z$ al wip $T 17$ manger $G I O$ gnvge $T(W)$ gnůgen die $U \quad 18$ h. mere $O Z$ vnd mere $L$. von S. sag. $G$ von S. tr. sag. IOLZ TUV 20 daz ist *G TUWFr26 - ob] daz $O$ * in] om. GI mich $L \quad$ im $W \quad 21$ laszen $L \quad$. der vil getriwe $m$. $G$ den vil getriuwen m. $T(U L) \quad 22$ helfekliche $F r 69(G L T U) V$ hvfslichen $O$ 23 lîp] pris $m \quad 24$ ouch] doch $W F r 26$. vmb mich $m \quad 26$ so maht du $I$ so maht $O$ dů můst $U$. wol str. pfl. $Z 27$ im ligent $I$. schlechte $W \quad 28$ ich sage dirs mit rechte $W \quad 29$ wortes. $U$ es worchte $W$

Abb. 1: Musteredition des Dreißigers 253 (Parzival-Projekt). 
$* \mathrm{D}$

254 dâr nâch der künec heizet Lac. daz swert oestêt oanz einen slac. am andern ez zevellet gar. wil dûz denne wider bringen dar, bestêt g. ${ }^{*} G W$ stât g. ${ }^{*} T \quad$ e. tac ${ }^{*} T$ ez wirt ganz von des wazzers trân.

wider om. ${ }^{*} m * G * T$

des] dem ${ }^{*} G$

dû muost des urspringes hân underem velse, ê in beschîne der tac. der selbe heizet Lac.

$\begin{array}{lll}\text { u. einem (ein } m) \text { v. }{ }^{*} m & \text { u. dem v. }{ }^{*} G \quad \text { von dem v. }{ }^{*} T \text {. in bescheine }{ }^{*} m\end{array}$ der s. brunne h. Lac ${ }^{*} m{ }^{*} G{ }^{*} T$ [ez besch. *G

Sint diu stucke niht verrêrt,

10 der si rehte zein ander kêrt, sô si der brunne machet naz, ganz und sterker baz wirt im valz und ecke sîn rehte $o m .{ }^{*} m$

vester ${ }^{*} T$

werdent $* T$ und vliesent niht diu mâl ir schîn.

15 daz swert bedarf wol segens wort. ich vürhte, diu hâstû lâzen dort. Hâts aber dîn muot gelernet, sô wehset und kernet immer sælden kraft bî dir.

20 lieber neve, geloube mir. sô muoz gar dienen dîner hant, swaz dîn lîp dâ wunders vant. ouch mahtû tragen schône immer sælden krône diu mâl niht ${ }^{*} G$

dû hâst diu 1. ${ }^{*} m$ diu habestû l. (gel. ${ }^{*} T L$ ) ${ }^{*} G{ }^{*} T$ hât es a. " $m$ hât si a. ${ }^{*} G{ }^{*} T$. dîn munt ${ }^{*} m{ }^{*} G{ }^{*} T$ an $\operatorname{dir}{ }^{*} G$

25 hôhe ob den werden. den wunsch ûf der erden hâstû volleclîche. niemen ist sô rîche, der gein dir koste mege hân, 30 hâstû vrâge ir reht getân.

sô mahtû ${ }^{*} G$ dû maht ouch ${ }^{*} T$

i. der s. (selben $m$ ) kr. " $m(V){ }^{*} G$

gewalteclîche ${ }^{*} G$

der vr. ${ }^{*} T(L)$

"D: D - "m: $m$ Fr69 (254.11-18)

${ }^{*} G$ : GI O L Z - ${ }^{*}$ T: $T U V W$ Fr26 (254.5-13, 27-30)

7 Initiale I 9 Majuskel D Initiale $O$ (unausg.) 17 Majuskel D 27 Initiale I

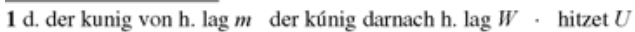
2 stat $T$ bestůnt $U$. gest. gang $m$ gest. ganz (von sp. Hand korrigiert aus gan:) $V$. e. $\operatorname{tac} T(U W) \quad 3$ Min and. $m$ - zủ vellet ez $L$ iz zu vellet $U$ zeruellet vil $W \quad \mathbf{4}$ wider om. ${ }^{*} m G I T \quad \mathbf{5}$ von des vaters tr. $m$ - des] dem GZ (Fr26) - tram $G$ stran UWFr26 7 vnder den brun v. $I$ von dem v. $T U$ von dirme v. Fr26 . ez besch. (beschein $O$ ) $G(O) L \quad 9$ stocke $U$, verseret $W \quad 10 \mathrm{zv}$ samne k. Z 11-13 Ganz vnd stercher baz / so si der brunne ie baz / machet im diu ekke $\sin I$. sterker] strecket $m$. heltze vnd egge s. $L 14$ div mal niht $G$ auch nih $I$. niht om. Fr69 15 senens w. $U \quad 16$ dv habst l. $O$. hastů $U$ - gelaszen Fr69 verlassen $W$ 17 hatz Fr69 hats $Z$. hat auer (aber $U$ ) si $I(U)$ aber om. $L \quad 18$ so wahset (verbessert aus gewurzet) $G$ - gernet $D \mathrm{mFr} 69$ (Z) TUV (in $V$ nachträglich verbessert $z u$ kernet) bernt $O$ vernet $L$ schermet $W \quad \mathbf{2 0}$ gloude bir $m$ nun volge mir $W \quad \mathbf{2 1} \mathrm{d}$. dir mein hand $W \quad 22$ dâ om. $L \quad 23.24$ so machtủ jemmer schone / tragen der salden crone $L$. tragen] targen $m$. i. der s. (selben $m$ ) kr. ${ }^{*} m V$ $G Z \quad 25$ ob] of $U \quad \mathbf{2 7}$ gewaltchliche $G(I O L) \quad 29$ kosten $m \quad 30$ der vr. $T(U) V(L)$

Abb. 2: Musteredition des Dreißigers 254 (Parzival-Projekt). 
*D

$\mathbf{4 5 4} E_{\mathrm{r}}$ was ein heiden vaterhalp, Flegetanis, der an ein kalp bette, als ob ez wære sîn got. wie mac der tievel sölhen spot 5 gevïegen an sô wîser diet, daz si niht scheidet ode schiet dâ von, der treit die hôhsten hant unt dem elliu wunder sint bekant? Flegetanis, der heiden,

10 kunde uns wol bescheiden ieslîches sternen hinganc unt sîner künfte widerwanc, wie lange ieslîcher umbe gêt, ê er wider an sîn zil gestêt.

15 mit der sternen umbereise vart ist geprüefet aller menneschlîcher art. ieglîches menschen art ${ }^{*} m(\mathrm{~V}) \quad$ a. mennischen art ${ }^{*} G \quad$ a. menschlîch art ${ }^{*} T$ Flegetanis, der heiden, sach, dâ von er blûclîche sprach, inme gestirne mit sînen ougen

20 verholenbariu tougen.

er jach, ez hiez ein dinc der Grâl. des namen las er sunder twâl inme gestirne, wie der hiez. »ein schar in ûf der erden liez,

25 diu vuor ûf über die sterne hôch, ob die ir unschult wider zôch. sît muoz sîn pflegen getouftiu vruht mit alsô kiuschlîcher zuht. diu menscheit ist immer wert,

30 der zuo dem Grâle wirt gegert. «
$\mathrm{Ez}{ }^{\star} G$

ob om. *G . sîn] ein ${ }^{*} m$

tiefel] vâlant ${ }^{*} T$. sînen sp. ${ }^{*} G * T$ uns om. ${ }^{*} m$

künste ${ }^{*} m{ }^{*} G(W)$

hine gêt *T

${ }^{*} D: D-{ }^{*} m: m$ [n: 454.17]

*G: GIOLZ - *T:TVW

1 Initiale D (unausg.) GIO (unausg.) L Z 17 Initiale I

$1 \mathrm{Ez} G I$ heiden om. $D \quad 3$ als wer es $W$. ob om. GIL · sîn] ein $m$ om. $L \quad 4$ tiefel] valant $T$. sinen sp. GIZTV $\mathbf{5}$ g. an do so w. $m$. gefóget $Z$ geru'gen $T$. werder d. $L \quad 7$ der do tr. $V$ der treg $W \quad \mathbf{8}$ den $m \cdot \sin Z \quad 12$ sinen $G \quad$. widerswanc $I$ wider vanc $Z \quad 13$ wie angeslich er $I \quad$. hine gêt $T \quad 15$ vmbkraiße $W 16$ ist gepvifel $D$. a. mennischen $\operatorname{art} G(I)$ a. menslicher $\operatorname{art} O(L Z)$ a. menslich art $T(W) \quad 17$ der meister $n \mathbb{Q} 18$ er] om. $L$ ê $T$. blwecliche $D$ blvchelichen $G(I O L T W)$ blv̂deklichen $V \quad 19 \mathrm{im}$ $\operatorname{den} G$ andem $O \quad \mathbf{2 0}$ verholn bere $m$ verholneberne $V$ verholne berre $W \quad 21$ er sprach $W$. ez wære $G(I O L){ }^{*} T \quad 22 \mathrm{~d}$. n. iach vnd las er $m$. erlazer $L \quad 23$ an dem g. $I$. das h. $W \quad 25$ uf om. $O W$ - vf vur die st. h. $I \quad 26$ ir om. $O 27$ must $I(O)$. getrówe vr. $V$ $\mathbf{2 8}$ kv́scher $(T) V(L) \quad \mathbf{3 0} \operatorname{div} T \quad$ ist $I \quad$ gewert $T$

Abb. 3: Musteredition des Dreißigers 454 (Parzival-Projekt). 
$501 D_{\text {în }}$ eheim gap dir ouch ein swert,

dâ mit dû sünden bist gewert,

sît daz dîn wol redender munt dâ leider niht tet vrâge kunt.

5 die sünde lâ bî den andern stên! wir ouch tâlanc ruowen gên!« wol geborner $\mathrm{m} .{ }^{*} \mathrm{~m}$ vrâgen ${ }^{*} m{ }^{*} G$ wir suln o. $\left({ }^{*} m\right){ }^{*} T$. dar umb t. slâfen gân $* m$ wênec wart in bette und kulter brâht. bett oder kultern wart in wênec br. ${ }^{*} m$. kultern und bette br. ${ }^{*} T$ si giengen êt ligen ûf ein bâht. daz leger was ir hôhen art in ein b. ${ }^{*} G{ }^{*} T$ ir om. "m

10 gelîche ninder dâ bewart. sus was er dâ vünfzehen tage. der wirt sîn pflac, als ich iu sage: krût unde wurzelîn, daz muose ir bestiu spîse sîn. wurze und krütelîn ${ }^{*} T$

15 Parzival die swære truoc durch süeze mare, wand in der wirt von sünden schiet unt im doch rîterlîchen riet. Eines tages vrâgt in Parzival:

20 »wer was ein man, lac vorme Grâl? der was al grâ bî liehtem vel.« der wirt sprach: »daz was Titurel. der selbe ist dîner muoter an. dem wart alrêst des Grâles van

25 bevolhen durch schermens rât. ein siechtuom, heizet pôgrât, treit er, die lem helfelôs. sîne varwe er iedoch nie verlôs, wand er den Grâl sô dicke siht, 30 dâ von er mac ersterben niht.

daz om. ${ }^{* T}$
der sw. ${ }^{*} m$
liebiu $m .{ }^{*} T$

Aber sprach dô P. ${ }^{*} T$. sprach P. ${ }^{*} m$ man, der lac *G*T Der wirt sprach: »daz ist Tyturel ${ }^{*} T$ der ist al grâ bî liehtem vel ${ }^{*} T$ ist] was ${ }^{*} G$ wart] was ${ }^{*} T$ schermes $* G\left({ }^{*} T\right)$ siechtage $*_{m} * T$ die leme treit (tet $m$ ) der h. ${ }^{*} m$ doch $* T$

mag er $*^{*} * G * T$

${ }^{*} D: D F r 11$ (501.1-11, 22-30) - " $m: m$

*G: GILZ - ${ }^{*}$ T: T V W O Fr39 (501.1-28)

1 Initiale D GILZO (unausg.) Versal T 7 Initiale Fr39 11 Majuskel $T 15$ Initiale I W (vorgez.) Versal T 19 Majuskel D Initiale $m$ Versal $T \quad 21$ Versal T

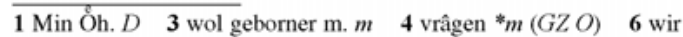
suln o. $\left({ }^{*} m\right)(I L Z) * T$. ouch $\mathrm{om}$. $W$. dar vmb talig sl. g. $m 7$ w. w. in beiden br. $I$. wênec] wen ich $L$ donoch Fr39 * in] im $Z$. kvtern $V$ kulter $W F r 39$ golter $O$. bet $W \mathbf{8}$ noch gulter si leiten sich in ein b. $I$. êt om. Frll . in ein b. Frll GIL T einen WFr39 9 ligen $I$. ir om. $m$. hơcheriv art Frll 10 dâ om. $O$ 11 dâ om. Fr39 13 wrcelin $D$ wirttelin $m$ wurzelen $V \quad 14$ mús $m$. ir beder sp. $I \quad 15$ din sw. $G$ div sw. OFr $3918 \mathrm{im}$ om. $O$ 19 sprach P. $m \quad 20$ man der lac GI TFr39 21.22 Der wirt sprach daz ist Tyturel / der ist al gra bi liehtem vel $T$ al om. $I \quad 23$ ist] was GIZ $\mathbf{2 4}$ dem wartet allez $I$ der w. alr. $L$ wart] was $T \mathbf{2 5}$ wan er wart im b. d. shermes rat $I$, bevolhens $D$, schermes $G(I L * T)$ 26 einen $O$. siechtage ${ }^{*} m(T) V$. der h. prograt $V \quad 28$ doch Frll $\mathrm{LL}{ }^{*} \mathrm{~T} 29$ dicke om. $W \quad 30$ (add. so $\mathrm{V}$ ) mag er Frll ${ }^{*} m * G$ $* T$

Abb. 4: Musteredition des Dreißigers 501 (Parzival-Projekt). 